\title{
Development and high-power testing of an $X$-band dielectric-loaded power extractor
}

\author{
Jiahang Shao $\odot,{ }^{1, *}$ Chunguang Jing, ${ }^{1,2}$ Eric Wisniewski, ${ }^{1}$ Gwanghui Ha $\odot,{ }^{1}$ Manoel Conde, ${ }^{1}$ \\ Wanming Liu, ${ }^{1}$ John Power, ${ }^{1}$ and Lianmin Zheng ${ }^{1, \dagger}$ \\ ${ }^{1}$ Argonne National Laboratory, Lemont, Illinois 60439, USA \\ ${ }^{2}$ Euclid Techlabs, Bolingbrook, Illinois 60440, USA
}

(Received 9 July 2019; published 3 January 2020)

\begin{abstract}
Dielectric-loaded structures are promising candidates for use in structure wakefield acceleration. They could be useful for both the collinear wakefield and the two-beam acceleration (TBA) approaches, due to their low fabrication cost, low radio-frequency (rf) losses, and potential to withstand high gradients. A short rf pulse ( $\leq 20 \mathrm{~ns}$ ) TBA program is currently being developed at the Argonne Wakefield Accelerator (AWA) facility, where dielectric-loaded structures are being used for both the power extraction and transfer structure (PETS) and the accelerator. In this study, an $X$-band $11.7 \mathrm{GHz}$ dielectric PETS was developed and tested at AWA to demonstrate high-power, short-pulse wakefield generation. The PETS was driven by a $1.3 \mathrm{GHz}$ train of eight electron bunches separated by $769.2 \mathrm{ps}$ ( 9 times the $X$-band rf period) in order to achieve coherent wakefield superposition. A total train charge of $360 \mathrm{nC}$ was passed through the PETS structure to generate $\sim 200 \mathrm{MW}, \sim 3 \mathrm{~ns}$ flat-top rf pulses without rf breakdown. A future experiment is being planned to increase the generated rf power to approximately $\sim 1 \mathrm{GW}$ by optimizing the structure design and improving the drive beam quality.
\end{abstract}

DOI: 10.1103/PhysRevAccelBeams.23.011301

\section{INTRODUCTION}

The advanced accelerator concepts (AAC) field conducts long-term research aimed at a future large-scale collider that will operate at substantially higher energy and lower cost than is possible with current accelerator technology [1-3]. Over the last several decades, four schemes for accomplishing this have been intensely investigated. Two are laser-driven: laser wakefield acceleration [4-6] and dielectric laser acceleration [7-9]. The other two are beamdriven: plasma wakefield acceleration [10-12] and structure wakefield acceleration (SWFA) [13-27].

In SWFA, a high-charge drive beam traveling through a structure excites wakefields, which are then used to accelerate a low-charge main beam, in either the same structure (collinear wakefield acceleration, CWA) or a parallel structure (two-beam acceleration, TBA) [3]. Based on our current understanding, the TBA approach is favored over CWA in a linear collider for two reasons. First, the beam transportation is less challenging because the beamline lattices for the main beam and the drive beam

\footnotetext{
*jshao@anl.gov

Visiting Argonne from Tsinghua University in Beijing.
}

Published by the American Physical Society under the terms of the Creative Commons Attribution 4.0 International license. Further distribution of this work must maintain attribution to the author(s) and the published article's title, journal citation, and DOI. can be optimized separately [3]. Second, the independent structures provide more flexibility to optimize the deceleration and acceleration parameters [22]. Currently, both the mature design of the Compact Linear Collider (CLIC) [28] and the AAC straw-man design of the Argonne Flexible Linear Collider (AFLC) [19,22] are based on the TBA approach. The major difference between the two colliders is that AFLC is designed with a shorter rf pulse ( $20 \mathrm{~ns}$, versus $230 \mathrm{~ns}$ in CLIC) with the expectation of achieving a higher accelerating gradient $(\sim 270 \mathrm{MV} / \mathrm{m}$, versus $100 \mathrm{MV} / \mathrm{m}$ in CLIC). This expectation is based on published evidence that the rf breakdown rate decreases with pulse length [29]. After optimizing structure and beam parameters, AFLC's efficiency with such short rf pulses is comparable to CLIC's [19,22].

In order to optimize the gradient, efficiency, and cost of a TBA-based collider, various novel accelerating/decelerating structures have been studied: metallic two-halves structure [21,30,31], dielectric-loaded structure (DLS) [13-16,20], metameterial structure [27,32,33], photonic band gap structure [34-36], and many others [17,24, 26,37-43]. Among them, the DLS is a very attractive candidate for a TBA-based collider due to its simple geometry, low fabrication cost (based on dimensions within the current limits of fabrication technology), high group velocity with reasonable shunt impedance, and potential to withstand $\mathrm{GV} / \mathrm{m}$ gradients $[20,44]$.

DLSs were first used for particle acceleration in the late 1940s, when they used lossy microwave-class glazed 
ceramics $[45,46]$. Since then, they have steadily improved. Current improvements include an increased frequency range from $\mathrm{GHz}$ to $\mathrm{THz}[13-16,20,47]$, comparable shunt impedance to the metallic disk-loaded structure by using low loss microwave ceramic materials $[15,22]$, tunability with a second layer of nonlinear ferroelectric [16], higher order modes (HOMs) damping with segmented conducting boundaries [48], multipacting suppression by external magnetic field $[49,50]$, and $\mathrm{GV} / \mathrm{m}$ level gradient in $\mathrm{rf}$ breakdown tests [44], as well as CWA experiments [20]. Despite this progress, high-power rf pulse generation beyond $100 \mathrm{MW}$ with drive bunch train excitation in the TBA approach had yet to be demonstrated for DLS. Previous results were limited to 30-50 MW due to the drive beam limitations as well as the immature design and fabrication methods of DLSs [15,22,51].

In this paper, we present the start-to-end development of an $X$-band $11.7 \mathrm{GHz}$ dielectric-loaded power extractor. This includes design optimization, simulation, fabrication, cold test, and high-power test. This work is an important step toward the realization of the short-pulse TBA-based future linear colliders as described in the AAC Roadmap [2]. It provides useful experimental information on $\mathrm{rf}$ breakdown in the nanosecond regime. Moreover, the start-to-end development presented here is generally applicable to other power extractors. The paper is organized as follows: Sec. II presents the structure design and simulation; Sec. III introduces the structure fabrication and the cold test; Sec. IV provides the high-power test setup, experimental results, and data analysis; Sec. V presents a future study toward gigawatt rf power generation; and Sec. VI summarizes the current study.

\section{DESIGN AND SIMULATION}

A power extraction and transfer structure consists of a decelerating structure and an rf coupler. In the decelerating structure, the drive beam transfers its energy via wakefields into the $\mathrm{TM}_{01}$ mode of the structure. Depending on the group velocity of the mode, the radiation moves either forward or backward with respect to the drive beam [27]. An rf coupler then extracts the $\mathrm{TM}_{01}$ mode from the decelerator and converts it into the $\mathrm{TE}_{10}$ mode of the rectangular waveguide which then transfers it to the accelerating structure.

The dielectric PETS used in this study (Fig. 1) consists of a cylindrical dielectric tube of uniform thickness with tapered dielectric sections at each end and an rf coupler at the output end. Deceleration of the drive beam takes place in the uniform section of the DLS [Fig. 1(b)] where the beam-structure interaction is strongest. Matching sections [Fig. 1(c)] are located at both ends of the uniform section for impedance matching between the uniform section and the vacuum pipe. The rf coupler extracts the power into the rectangular waveguide. In general, the PETS can be designed as a uniform tube without the tapered

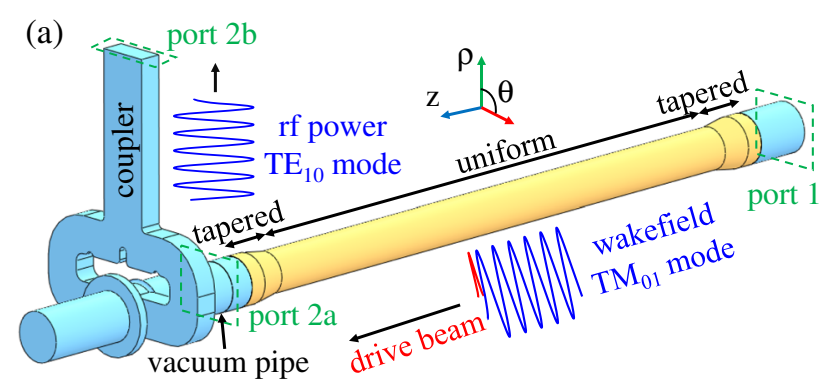

(b)

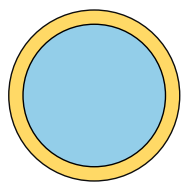

(c)

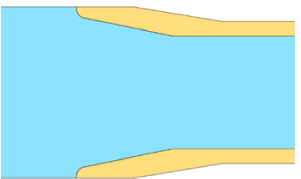

FIG. 1. Dielectric-loaded power extractor. Yellow and blue denote the dielectric and vacuum regions, respectively. The copper, which covers the outside of the whole structure, is not shown. The drive beam travels through the center of the dielectric tube along the $z$-axis. (a) Layout of the whole structure. (b) Transverse cross section of the uniform dielectric section. (c) Longitudinal cross section of the tapered dielectric matching section.

sections [15,51], a segmented tube with different impedance sections [52], or in one of several other ways.

In the remainder of this section, we present the detailed design and simulation of the uniform deceleration section, the matching section, and the rf coupler.

\section{A. Uniform section}

The design of the uniform deceleration section determines the strength and the duration of the generated $\mathrm{rf}$ power under certain drive beam conditions. In the design phase, the geometry is optimized based on considerations such as the required rf power level and pulse length, drive beam transportation, availability of dielectric materials, and fabrication restrictions $[15,22]$. The design procedure can be divided into two parts: electromagnetic (EM) properties of the structure and rf pulse properties. The assumptions used in the derivation of this section are as follows: (1) Only the cylindrical $\mathrm{TM}_{01}$ mode is taken into consideration (for simplicity). (2) The electron beam is ultrarelativistic. (3) The longitudinal bunch profile follows the Gaussian distribution.

\section{1. $\mathrm{TM}_{01}$ mode properties}

In this subsection, we solve for the EM field properties of the $\mathrm{TM}_{01}$ accelerating mode, its dispersion relation, and several key figures of merit. We begin by assuming the mode propagates without attenuation, but we will include it at the end of this subsection.

The general $\mathrm{TM}_{01}$ propagation equations in the dielectric-loaded structure are given by 


$$
\left\{\begin{array}{l}
T_{1}^{2}+\beta^{2}=\omega_{0}^{2} \epsilon_{0} \mu_{0}=\frac{\omega_{0}^{2}}{c^{2}} \\
T_{2}^{2}+\beta^{2}=\omega_{0}^{2} \epsilon_{0} \epsilon_{r} \mu_{0}
\end{array}\right.
$$

where the subscripts 1 and 2 denote the vacuum region and the dielectric region, respectively; $T_{1,2}$ is the cutoff wave number; $\beta$ is the propagation constant in the $\mathrm{z}$ direction; $\epsilon_{0}$ is the vacuum permittivity; $\epsilon_{r}$ is the dielectric constant; $\mu_{0}$ is the vacuum permeability; $\omega_{0}$ is the frequency; and $c$ is the speed of light.

In the $\mathrm{TM}_{01}$ mode, $E_{\theta}=0, H_{\rho}=0$, and $H_{z}=0$. The other field components can be expressed as (ignoring the time and longitudinal position dependent term $e^{j\left(\omega_{0} t-\beta z\right)}$ )

$$
\left\{\begin{array}{l}
E_{\rho, 1}=j \beta T_{1} A J_{1}\left(T_{1} \rho\right) \\
E_{z, 1}=T_{1}^{2} A J_{0}\left(T_{1} \rho\right) \\
H_{\theta, 1}=j \omega_{0} \epsilon_{0} T_{1} A J_{1}\left(T_{1} \rho\right) \\
E_{\rho, 2}=j \beta T_{2}\left[B J_{1}\left(T_{2} \rho\right)+C N_{1}\left(T_{2} \rho\right)\right] \\
E_{z, 2}=T_{2}^{2}\left[B J_{0}\left(T_{2} \rho\right)+C N_{0}\left(T_{2} \rho\right)\right] \\
H_{\theta, 2}=j \omega_{0} \epsilon_{0} \epsilon_{r} T_{2}\left[B J_{1}\left(T_{2} \rho\right)+C N_{1}\left(T_{2} \rho\right)\right]
\end{array},\right.
$$

where $A, B$, and $C$ are constants that can be determined by the boundary conditions and excitation strength; $J_{n}$ and $N_{n}$ are the $n$ th-order Bessel functions of the first and the second kinds, respectively.

The boundary conditions are given by

$$
\left\{\begin{array}{l}
\left.E_{z, 1}\right|_{\rho=a}=\left.E_{z, 2}\right|_{\rho=a} \\
\left.H_{\theta, 1}\right|_{\rho=a}=\left.H_{\theta, 2}\right|_{\rho=a}, \\
\left.E_{z, 2}\right|_{\rho=b}=0
\end{array}\right.
$$

where $a$ and $b$ denote the inner and the outer radius of the tube respectively.

From the preceding equations, the dispersion relation can be derived as

$$
\frac{T_{2} J_{1}\left(T_{1} a\right)}{\epsilon_{r} T_{1} J_{0}\left(T_{1} a\right)}=\frac{J_{1}\left(T_{2} a\right) N_{0}\left(T_{2} b\right)-J_{0}\left(T_{2} b\right) N_{1}\left(T_{2} a\right)}{J_{0}\left(T_{2} a\right) N_{0}\left(T_{2} b\right)-J_{0}\left(T_{2} b\right) N_{0}\left(T_{2} a\right)}
$$

Given the propagation equation [Eq. (1)] and the dispersion relation [Eq. (4)] just derived, we can now choose the DLS parameters such that the phase velocity $\left(v_{p}=\omega_{0} / \beta\right)$ of the cylindrical $\mathrm{TM}_{01}$ mode is equal to the beam velocity, as needed for synchronization. This is accomplished by solving Eqs. (1) and (4) for $b$, given a set of $a, \varepsilon_{r}, \omega_{0}$, and $\beta=\omega_{0} / c$.

Given this set of parameters $\left(a, b, \epsilon_{r}\right.$, and $\left.\omega_{0}\right)$, we can now calculate several key figures of merit of the $\mathrm{TM}_{01}$ mode in the DLS.

The group velocity $\left(v_{g} \equiv \beta_{g} c=d \omega_{0} / d \beta\right)$ is found by varying $\beta$ as a function of $\omega_{0}$ and using Eqs. (1) and (4).
The stored energy is given by

$$
\begin{aligned}
U= & \frac{1}{2} \epsilon_{0} \int_{V_{1}}\left(\left|E_{\rho, 1}\right|^{2}+\left|E_{z, 1}\right|^{2}\right) d v \\
& +\frac{1}{2} \epsilon_{0} \epsilon_{r} \int_{V_{2}}\left(\left|E_{\rho, 2}\right|^{2}+\left|E_{z, 2}\right|^{2}\right) d v,
\end{aligned}
$$

where $V_{1}$ and $V_{2}$ denote the volume of the vacuum and the dielectric respectively.

The attenuation of the $\mathrm{TM}_{01}$ mode in the DLS is due to losses in both the dielectric $P_{\text {diel }}$ and the copper wall $P_{\text {wall }}$, which can be calculated as

$$
\left\{\begin{array}{l}
P_{\text {diel }}=\frac{\omega_{0} \epsilon_{0} \epsilon_{r} \tan \left(\delta_{d}\right)}{2} \int_{V_{2}}\left(\left|E_{\rho, 2}\right|^{2}+\left|E_{z, 2}\right|^{2}\right) d v \\
P_{\text {wall }}=\left.\frac{1}{2 \sigma \delta} \oint_{S}\left|H_{\theta, 2}\right|_{\rho=b}\right|^{2} d s
\end{array},\right.
$$

where $S$ denotes the surface between copper and dielectric; $\sigma$ is the conductivity of copper; $\delta=\sqrt{2 /(\omega \mu \sigma)}$ is the skin depth; and $\tan \left(\delta_{d}\right)$ is the loss tangent of the dielectric material.

Finally, the quality factor $Q$ and the shunt impedance per unit length $r$ of the uniform DLS section are given by

$$
\left\{\begin{array}{l}
Q=\frac{\omega_{0} U}{P_{\text {wall }}+P_{\text {diel }}} \\
r=\frac{\left(\left.\int_{s}^{L_{s t}} E_{z, 1}\right|_{\rho_{0}} d l\right)^{2}}{\left(P_{\text {wall }}+P_{\text {diel }}\right) L_{s t}},
\end{array}\right.
$$

where $L_{s t}$ is the length of the uniform section.

Three low-loss dielectric materials with $\tan \left(\delta_{d}\right)=1 \times$ $10^{-4}$ were considered for the design: quartz $\left(\epsilon_{r}=3.75\right)$, alumina $\left(\epsilon_{r}=9.8\right)$, and $\mathrm{MTO}\left(\mathrm{MgTiO}_{3}-\mathrm{Mg}_{2} \mathrm{TiO}_{4}, \epsilon_{r}=\right.$ 16) [53]. When the power extractor operates at $11.7 \mathrm{GHz}$ and is synchronized to an ultrarelativistic drive beam, we
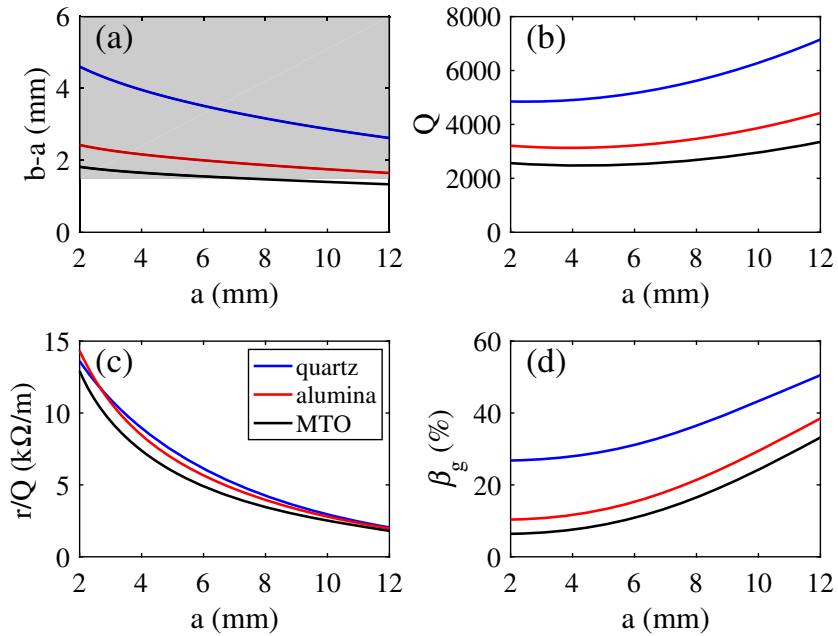

FIG. 2. Properties of the uniform DLS section as a function of the inner radius for different dielectric materials. (a) Dielectric wall thickness. (b) $\mathrm{TM}_{01}$ quality factor. (c) $\mathrm{TM}_{01} r / Q$. (d) $\mathrm{TM}_{01}$ group velocity. 
have numerically calculated the EM properties as a function of the inner radius (Fig. 2).

\section{2. rf power generated by the beam-driven DLS}

In this subsection, we first derive the rf parameters due to a single (subscript "s") drive bunch and then the rf parameters due to multiple (subscript "m") drive bunches (i.e., a drive bunch train).

When an ultrarelativistic drive bunch passes through the uniform section of the DLS $\left(v_{g}>0\right)$, the leading edge of its wakefield packet travels together with the bunch while the trailing edge moves forward at the group velocity [15,22]. Therefore, the rf pulse length of the generated wakefield packet at the end of the DLS is given by

$$
\tau=\frac{L_{s t}}{v_{g}}-\frac{L_{s t}}{c}=\frac{L_{s t}\left(1-\beta_{g}\right)}{c \beta_{g}} .
$$

The longitudinal electric field of the generated wakefield at the end of the DLS, including structure attenuation, is $(0 \leq t \leq \tau)$

$$
E_{S}(t)=\left|E_{s}(t)\right| \cos \left(\omega_{0} t\right)=2 \Phi Q_{b} H(c t) \kappa_{l} e^{-\frac{\alpha c \beta_{g} t}{1-\beta_{g}}} \cos \left(\omega_{0} t\right),
$$

where $Q_{b}$ is the bunch charge; $\Phi=\exp \left[-\left(\omega_{0} \sigma_{z} / c\right)^{2} / 2\right]$ is the form factor; $\sigma_{z}$ is the root-mean-square (rms) bunch length; $\kappa_{l}=\left(\omega_{0} / 4\right)(r / Q)\left[1 /\left(1-\beta_{g}\right)\right]$ is the loss factor per unit length [54]; $\alpha=\omega_{0} /\left(2 Q v_{g}\right)$ is the attenuation factor of the structure; and $H$ is the Heaviside step function. The time, $t=0$, is set when the drive beam reaches the exit of the uniform section.

The spectrum of the generated wakefield, given by the Fourier transform of Eq. (9) after ignoring structure attenuation, is

$$
F_{s}(\omega)=D \operatorname{sinc}\left[\frac{\tau\left(\omega-\omega_{0}\right)}{2}\right],
$$

where $D$ is a constant.

The average energy loss of the bunch, by integrating the experienced field over the entire structure, is

$$
U_{\text {loss }}=\Phi \int_{0}^{L_{s t} / c} c E_{s}(0) d t=\Phi^{2} Q_{b} \kappa_{l} L_{s t} .
$$

The generated $\mathrm{rf}$ power at end the end of the DLS, following the general relation of $P=E^{2} v_{g} /(\omega r / Q)$ in traveling-wave structures, can be expressed as

$$
P_{s}(t)=\frac{c \beta_{g}\left[E_{s}(t)\right]^{2}}{4 \kappa_{l}\left(1-\beta_{g}\right)} .
$$

When a multibunch train of $N$ bunches separated by $T_{b}$ passes through the uniform section, the rf fields from each bunch superpose to give
$E_{m}(t)=\sum_{k=1}^{N} E_{s, k}\left[t-(k-1) T_{b}\right] \equiv\left|E_{m}(t)\right| \cos (\omega t)$,

where $E_{s, k}$ is the longitudinal electric field excited by the $k$ th bunch.

In this case, the spectrum of the generated wakefield, given by the Fourier transform of Eq. (13) after ignoring structure attenuation, is

$$
F_{m}(\omega)=\sum_{k=1}^{N} F_{s, k}(\omega) e^{-j(k-1) \omega T_{b}},
$$

where $F_{s, k}$ is the spectrum excited by the $k$ th bunch.

The average energy loss of the $k$ th bunch in the train, by integrating its experienced field over the entire structure, is

$$
U_{\mathrm{loss}, k}=\Phi \int_{0}^{L_{s t} / c} c E_{m}\left[(k-1) T_{b}\right] d t
$$

Due to the finite length of the rf pulse excited by a single bunch, $E_{m}$ reaches its maximum value after a rise time due to $N_{\text {rise }}=\left\lceil\tau / T_{b}\right\rceil-1$ bunches. For the rf pulse excited by $N$ bunches, the rise time $t_{\text {rise }}$, the flat-top duration $t_{\text {flat }}$, and the fall time $t_{\text {fall }}$ can be expressed as

$$
\left\{\begin{array}{l}
t_{\text {rise }}=N_{\text {rise }} T_{b} \\
t_{\text {flat }}=\left(N-N_{\text {rise }}\right) T_{b} . \\
t_{\text {fall }}=\tau-T_{b}
\end{array}\right.
$$

When the drive bunches are spaced at integer wavelengths, $T_{b}=2 \pi n / \omega_{0}, E_{s, k}$ adds coherently to yield the maximum possible amplitude of the multibunch field

$$
\left|E_{m}(t)\right|=\sum_{k=1}^{N}\left|E_{s, k}\left[t-(k-1) T_{b}\right]\right| .
$$

The average field amplitude of the flat-top can be expressed as

$$
\overline{E_{\text {flat }}}=\frac{\int_{N_{\text {rise }} T_{b}}^{N T_{b}}\left|E_{m}(t)\right| d t}{t_{\text {flat }}} \propto Q_{b} .
$$

Therefore, the average power of the flat-top, following $P=E^{2} v_{g} /(\omega r / Q)$, is

$$
\overline{P_{\text {flat }}}=\frac{c \beta_{g}{\overline{E_{\text {flat }}}}^{2}}{4 \kappa_{l}\left(1-\beta_{g}\right)} \propto Q_{b}^{2} .
$$

For a given set of $T_{b}, N, Q_{b}$, and $\sigma_{z}, \overline{P_{\text {flat }}}$ can be increased by using longer structures at the expense of a shorter rf flat-top duration, as illustrated in Fig. 3. Therefore, a long uniform section is chosen in this study in order to maximize the rf power level. Its length is fixed at 


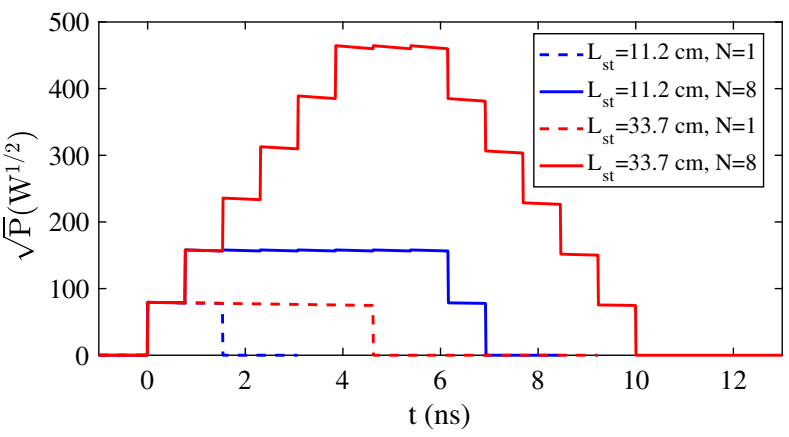

FIG. 3. Calculated square root of the output power using the structure parameters listed in Table I but with various lengths. In the calculation, $T_{b}=769.2 \mathrm{ps}, Q_{b}=1 \mathrm{nC}$, and $\sigma_{z}=1.5 \mathrm{~mm}$.

$26 \mathrm{~cm}$, which is the practical limit of the current fabrication technology for a single dielectric tube. This length of dielectric tube restricts the wall thickness to be greater than $1.5 \mathrm{~mm}$ in order to prevent it from cracking during fabrication. This restriction is indicated as the shaded region in Fig. 2(a).

Given the fixed uniform section length of $26 \mathrm{~cm}, t_{\text {flat }}$ and $\overline{P_{\text {flat }}}$ of the structures illustrated in Fig. 2 can be numerically calculated based on the actual drive bunch parameters available at AWA: $T_{b}=769.2 \mathrm{ps}(1.3 \mathrm{GHz}$ or the 9th subharmonic of the $X$-band frequency), $N=8$, and $Q_{b} \leq$ $60 \mathrm{nC}$ [55]. Figure 4 illustrates the results for $Q_{b}=30 \mathrm{nC}$ and $\sigma_{z}=1.5 \mathrm{~mm}$, which are typical for routine operation. The design goals are to have a flat-top duration longer than $2 \mathrm{~ns}$ and an average power greater than $100 \mathrm{MW}$. The shaded regions indicate where the design goals are satisfied.

Accounting for both the EM and rf generation properties (Fig. 2 and Fig. 4, respectively) of the DLS, we can now find the values of inner radius and materials that satisfy our design goals. These are found to be: $a \leq 6.3 \mathrm{~mm}$ for quartz; $6.2 \mathrm{~mm} \leq a \leq 7.9 \mathrm{~mm}$ for alumina; and no proper $a$ for MTO. Given the difficulty of transmitting a high charge drive bunch train through the DLS, a larger inner radius is preferred. Therefore, alumina has been chosen with $a$ set to $7.50 \mathrm{~mm}$. The detailed parameters of the uniform section used in this study are listed in Table I.
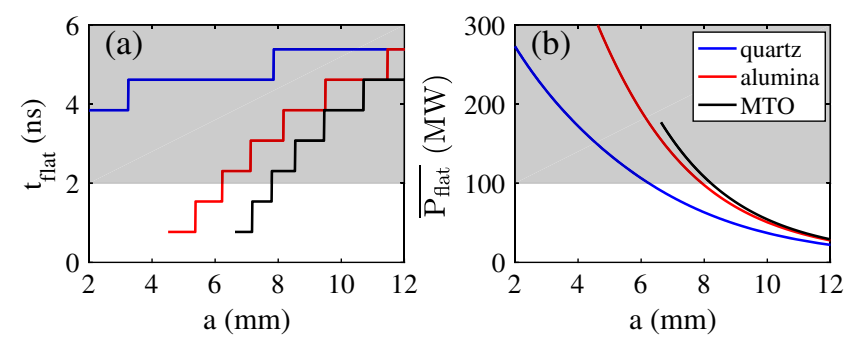

FIG. 4. Duration (a) and average power (b) of the flat-top as a function of the inner radius for different dielectric materials.
TABLE I. Parameters of the uniform section.

\begin{tabular}{lc}
\hline \hline Parameter & Value \\
\hline Dielectric material & Alumina \\
Dielectric constant & 9.8 \\
Loss tangent & $1 \times 10^{-4}$ \\
Inner radius & $7.50 \mathrm{~mm}$ \\
Outer radius & $9.40 \mathrm{~mm}$ \\
Length & $26 \mathrm{~cm}$ \\
Outer boundary material & Copper \\
Outer boundary conductivity & $5.8 \times 10^{7} \mathrm{~S} / \mathrm{m}$ \\
Quality factor & 3393 \\
$r / Q$ & $4.32 \mathrm{k} \Omega / \mathrm{m}$ \\
Group velocity & $0.20 \mathrm{c}$ \\
$E_{\text {surface,max }} / E_{\text {axis }}$ & 1 \\
Flat-top duration $(8-b u n c h$ train) & $3.1 \mathrm{~ns}$ \\
\hline \hline
\end{tabular}

\section{B. Matching section and coupler}

An rf coupler is installed at the end of the uniform section to convert the $\mathrm{TM}_{01}$ mode of the circular DLS into the $\mathrm{TE}_{10}$ mode of the rectangular WR-90 waveguide. In previous studies of dielectric PETS, the rf output coupler was customized and could not be reused for other structures $[15,51]$. To simplify the design and reduce cost, a broadband dual-feed coupler has been modified from the $11.424 \mathrm{GHz}$ version broadly used in $X$-band metallic structure high-gradient research [56,57].

A dielectric matching section is used to match the impedance between the uniform dielectric section (inner diameter of $15.0 \mathrm{~mm}$ ) and the rf coupler (vacuum pipe diameter of $22.7 \mathrm{~mm}$ ) at the beam exit, as illustrated in Fig. 1(c). An adiabatic matching section with a gradual dielectric taper is used to achieve a wide band width for short rf pulses. Optimal results were achieved by adjusting the length and angle of the dielectric tapered section, as illustrated in Fig. 5 (simulation with CST Microwave Studio [58]). The results have also been verified with the ACE3P code [59]. For the entire structure, including the coupler, we achieved $\mathrm{S}_{21}$ at $11.7 \mathrm{GHz}$ of $-0.7 \mathrm{~dB}$ with $3 \mathrm{~dB}$ bandwidth of over $1 \mathrm{GHz}$. The peaks separated by $\sim 90 \mathrm{MHz}$ in the $S_{11}$ curve are caused by the matching section design.

The same dielectric tapered section is also designed at the beam entrance side to reduce the transition radiation between the vacuum pipe and the uniform section. The total length of the DLS plus the matching sections at both ends is $30 \mathrm{~cm}$. The length of the entire structure, including the rf coupler, is $42 \mathrm{~cm}$.

The rf output from the dielectric PETS, shown at the coupler port (Fig. 1, port 2b) when driven by a single bunch, is shown in Fig. 6 (simulation with CST Particle Studio, wakefield module [58]). The length and amplitude are in good agreement with the analytic estimates based on Eqs. (8) and (12), which only take the uniform section into consideration. In the CST simulation results, the rising time 


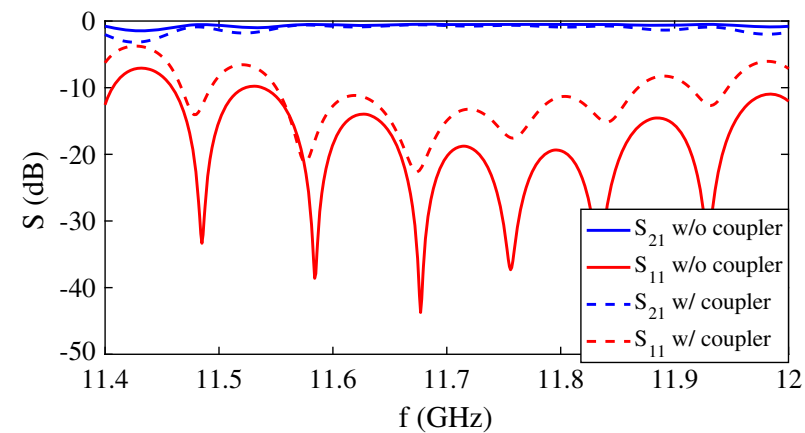

FIG. 5. Simulated S-parameters of the structure. The port definition is illustrated in Fig. 1: port 1 at the beam entrance; port 2 at the beam exit when simulated without the coupler (port $2 \mathrm{a})$ or at the waveguide port when simulated with the coupler (port 2b).

is caused by the finite bandwidth of the structure; the falling time is a result of the bandwidth and the transient process of the wakefield entering the downstream vacuum pipe [60]. Finally, the non-ideal flatness should be caused by a small reflection that arose from a slight impedance mismatch between the uniform section and the rf coupler, or beating from modes excited near the matching section or the $\mathrm{rf}$ coupler. These effects are not caused by the uniform section, as verified by the good flatness when simulating only the uniform section without the matching sections nor the rf coupler [see Fig. 1(b), where the output port is defined at the beam exit of the uniform cylindrical tube], as illustrated in the inset of Fig. 6.

When driven by a multibunch train separated by $769.2 \mathrm{ps}$ $\left(T_{b}=2 \pi / \omega_{0} \times 9\right)$, the port signal is coherently enhanced, as illustrated in Fig. 7. The flat-top of the rf pulse is reached at the 5th drive bunch and has a duration of $\sim 3 \mathrm{~ns}$. The average field amplitude and rf power of the flat-top driven by the 8-bunch train are 4.6 and 21.4 times of those driven by a single bunch. The full widths at half maximum (FWHM) of the frequency spectrum driven by a single

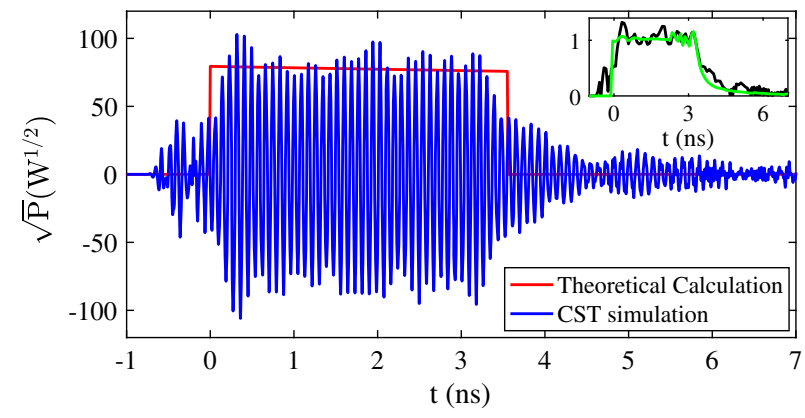

FIG. 6. Coupler output signal driven by a single bunch with $Q_{b}=1 \mathrm{nC}$ and $\sigma_{z}=1.5 \mathrm{~mm}$. The time, $t=0$, is set at the start of the analytically calculated rf pulse from Eq. (12). Inset: Comparison of the normalized output signal amplitude when simulating the entire structure (black) and only the uniform section without the matching sections nor the rf coupler (green).
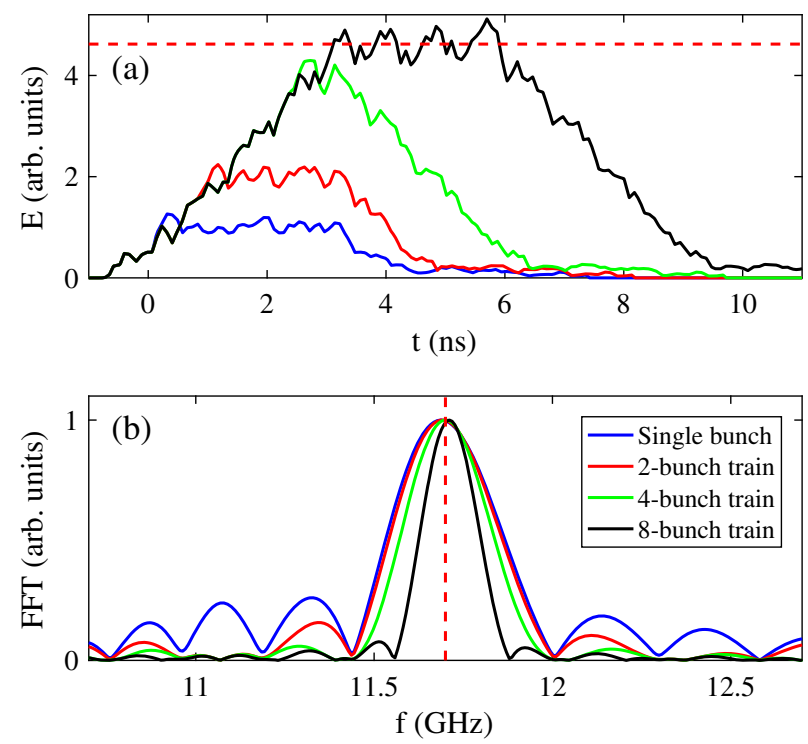

FIG. 7. Envelop (a) and spectrum (b) of the coupler output rf signal. For bunch trains, the field is calculated by summing those driven by a single bunch with proper delays. The field amplitude is normalized to that driven by a single bunch. The horizontal and vertical red dashed lines indicate the average amplitude of the flat-top driven by the 8 -bunch train and $f=11.7 \mathrm{GHz}$, respectively.

bunch, a 2-bunch train, a 4-bunch train, and an 8-bunch train are $0.34,0.32,0.27$, and $0.18 \mathrm{GHz}$ respectively; all of which are in good agreement with the values calculated by Eq. (14).

\section{FABRICATION AND COLD-TEST}

\section{A. Fabrication}

The mechanical layout of the $X$-band dielectric-loaded power extractor is shown in Fig. 8.

The dielectric tube with a metallic coating is fabricated as follows. The dielectric tube, including the uniform and tapered sections, was sintered as a single piece with a surface roughness of $\sim 100 \mathrm{~nm}$ by grinding. The metallic boundary consists of three layers. A chromium buffer layer of $\sim 10 \mathrm{~nm}$ was first sputtered onto the dielectric's outer surface, followed by a $\sim 100 \mathrm{~nm}$ thin copper layer sputtered onto the chromium. Finally a thick copper layer of $\sim 75 \mu \mathrm{m}$ was electroplated onto the thin copper layer, as illustrated in Fig. 8(a). Although chromium (conductivity of $7.9 \times 10^{6} \mathrm{~S} / \mathrm{m}$ ) is more lossy than copper, the rf loss on the chromium buffer layer is negligible due to its small thickness. The detailed derivation of the rf loss on a thin layer is presented in the Appendix. Therefore, the thin buffer layer was not considered in CST simulations, which also reduced the mesh number and the simulation time.

The entire power extractor/transfer structure was assembled as follows: (1) The output coupler and a center flange were connected. The electrical contact and the 


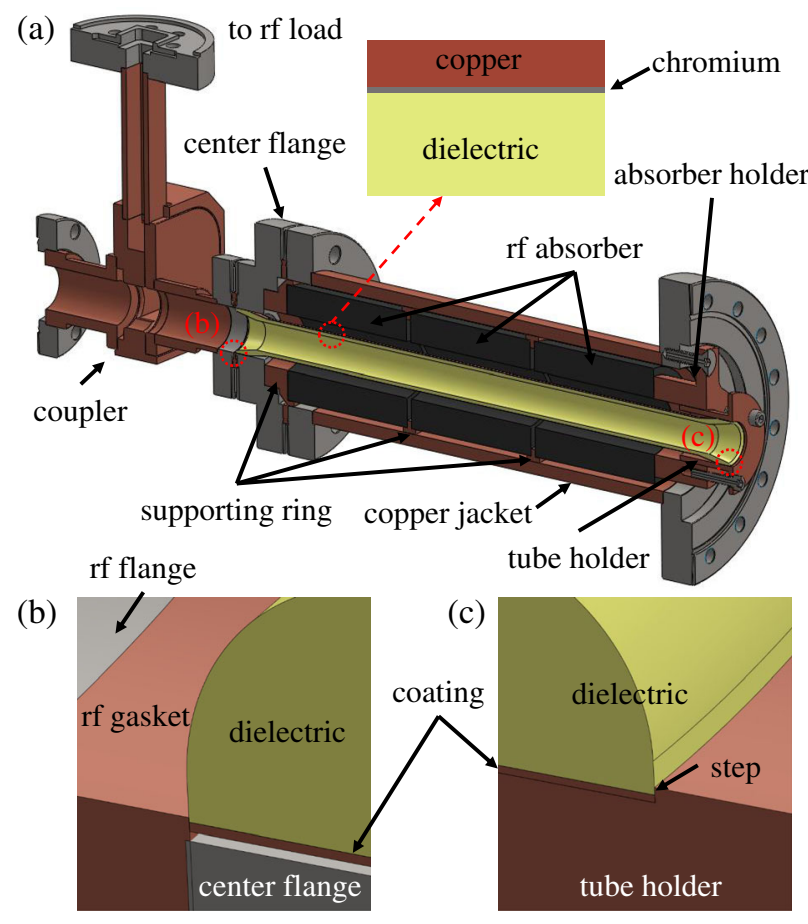

FIG. 8. (a) Cross-section of the entire power extractor/transfer structure assembly with details of the 3-layer metallic coating of the dielectric tube. (b) Magnified view of the contact between the dielectric tube and the rf gasket at the beam exit. (c) Magnified view of the contact between the dielectric tube and the tube holder at the beam entrance.

vacuum seal are ensured by a high-power rf gasket in between. (2) This assembly and a copper jacket [61] were connected. The electrical contact and vacuum seal are ensured by a regular gasket in between. (3) Three rf absorbers and their supporting rings were loaded into the chamber. The rings have several openings (not shown) to avoid trapped air when pumping. (4) The rf absorbers and the supporting rings were pushed against the center flange by an absorber holder that was screwed onto the chamber. (5) The coated dielectric tube was inserted into the chamber until it came to a stop against the high-power rf gasket. Details of the contact between the tube and the rf gasket are illustrated in Fig. 8(b). (6) The tube was held firmly against the rf gasket by a tube holder that screwed onto the absorber holder. The electrical contact between the tube and the tube holder is ensured by tight tolerance and a small step machined on the tube holder, as detailed in Fig. 8(c).

It should be noted that, during this experiment, the rf absorbers were only used to mechanically support the dielectric tube. However, in future studies, they will be used to damp HOMs using structures with segmented conducting boundaries [48]. In addition, no cooling was needed because the average output power was on the order of a watt due to the low machine repetition rate $(2 \mathrm{~Hz})$ used during the experiment.

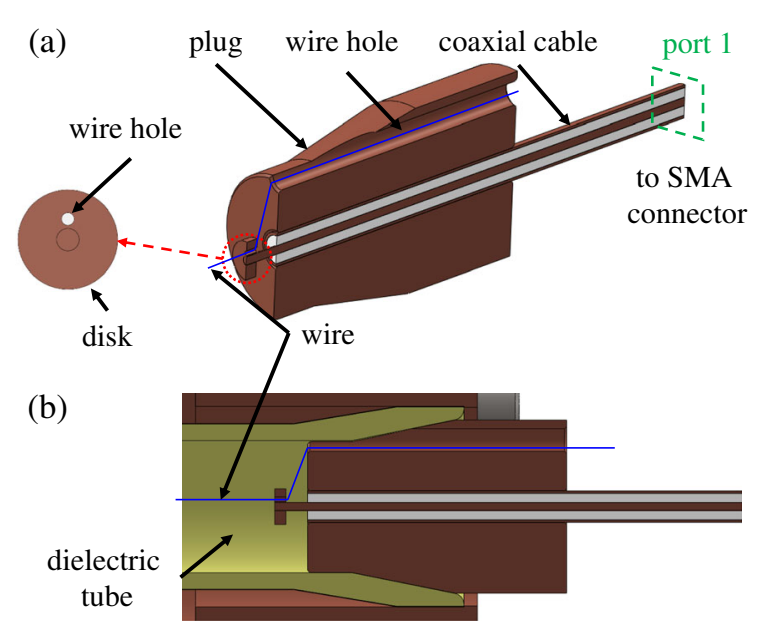

FIG. 9. (a) Mode launcher used during the cold test together with a magnified view of the disk. (b) Insertion of the mode launcher into the dielectric-loaded power extractor assembly.

\section{B. Cold test}

A mode launcher, illustrated in Fig. 9, was developed for the cold test because although the structure has an $\mathrm{rf}$ coupler at one end, it is open at the other. The launcher converts the TEM mode in the coaxial rf cable into the cylindrical $\mathrm{TM}_{01}$ mode of the dielectric PETS.

The assembly procedure of the mode launcher is as follows: (1) A $50 \Omega$ rigid coaxial rf cable was inserted into a plug that could fit tightly against the inner surface of the tapered dielectric section. The electrical contact between the cable and the plug is ensured by tight tolerance. (2) A disk with a small offset hole was welded onto the inner conductor of the rf cable. (3) A nylon wire used for the bead-pull (blue line in Fig. 9) was first passed through the hole in the disk and then through the one in the plug. (4) The metallic bead was made by tightly wrapping a short piece of metallic tape ( $\sim 1 \mathrm{~mm}$ long, not shown) onto the nylon wire. Note that the bead was slightly off-axis inside the dielectric tube due to the offset hole in the disk. However, the influence of this small offset $(0.8 \mathrm{~mm})$ during the bead-pull measurement [62] is negligible according to the CST Microwave Studio simulation.

\section{S-parameter}

A N5230C network analyzer was used for the cold test measurements. Port 1 was connected to the SMA connector of the mode launcher (port 1 in Fig. 9) and port 2 was connected to the waveguide output port of the rf coupler (port $2 \mathrm{~b}$ in Fig. 1) through a commercial SMA-to-WR90 adapter. The complete setup, including the fully assembled dielectric PETS and the mode launcher while excluding the SMA-to-WR90 adapter, was simulated with CST Microwave Studio. The simulated values of $S_{21}$ and $S_{11}$ at $11.7 \mathrm{GHz}$ are $-1.0 \mathrm{~dB}$ and $-21.3 \mathrm{~dB}$, respectively. This 


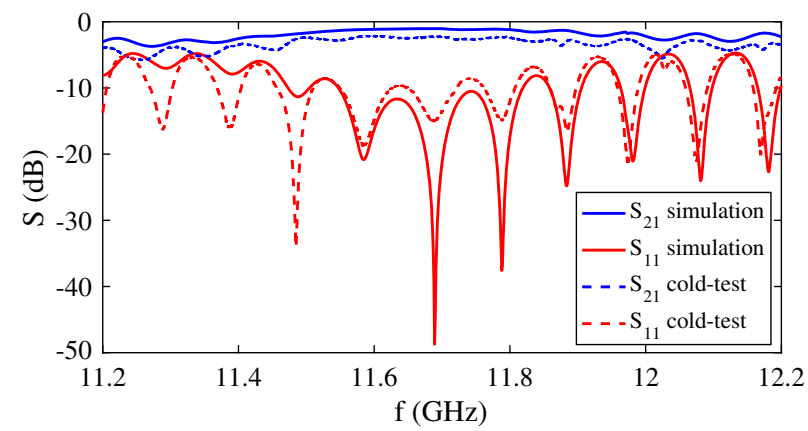

FIG. 10. Comparison of S-parameters between the cold-test measurements and the CST simulations.

shows good power coupling and transmission with board bandwidth, as indicated by the solid lines in Fig. 10.

The cold-test measurements are in reasonable agreement with the simulation, as indicated by the dashed lines in Fig. 10. The cold-test values of $S_{21}$ and $S_{11}$ at $11.7 \mathrm{GHz}$ are $-2.3 \mathrm{~dB}$ and $-13.6 \mathrm{~dB}$, respectively. These are slightly worse than the expected values from the simulation. The lower transmission $(2.3-1.0=1.3 \mathrm{~dB})$ can be caused by several factors, including higher if loss on the metallic wall of the structure $\left(P_{\text {wall }}\right)$ due to increased surface roughness from sputtering; rf losses in the SMA connector of the mode launcher and the SMA-to-WR90 adapter, which could not be calibrated; and imperfect electrical contact between the mode launcher and the tapered section due to machining tolerance.

\section{Field distribution}

The on-axis electric field was measured by the bead-pull method [62] and its amplitude and phase are illustrated by the solid blue lines in Fig. 11. The bead-pull results are in reasonable agreement with the CST Microwave Studio simulations shown by the solid red lines in Fig. 11.

The structure attenuation was calculated from the field amplitude distribution for both the bead-pull measurement and the CST simulation. The field amplitude in the uniform section follows

$$
E(z)=E_{0} \exp (-\alpha z) \approx E_{0}(1-\alpha z),
$$

from which the total rf loss of the uniform section is given by $20 \log _{10}\left[\exp \left(-\alpha L_{s t}\right)\right]$. The oscillation of the field amplitude in the uniform section is caused by the imperfect impedance matching and appears in both the bead-pull measurement and the CST simulation. To calculate $\alpha$, the field oscillation is ignored and the field is approximated by a linear fit.

In Fig. 11(a), the linear fits to the CST Microwave Studio simulation results and the cold-test results are shown by the green line and the black line, respectively. For the green line, the fitted $\alpha$ of $0.18 \mathrm{~m}^{-1}$ agrees well with the nominal value calculated using the parameters listed in Table I.
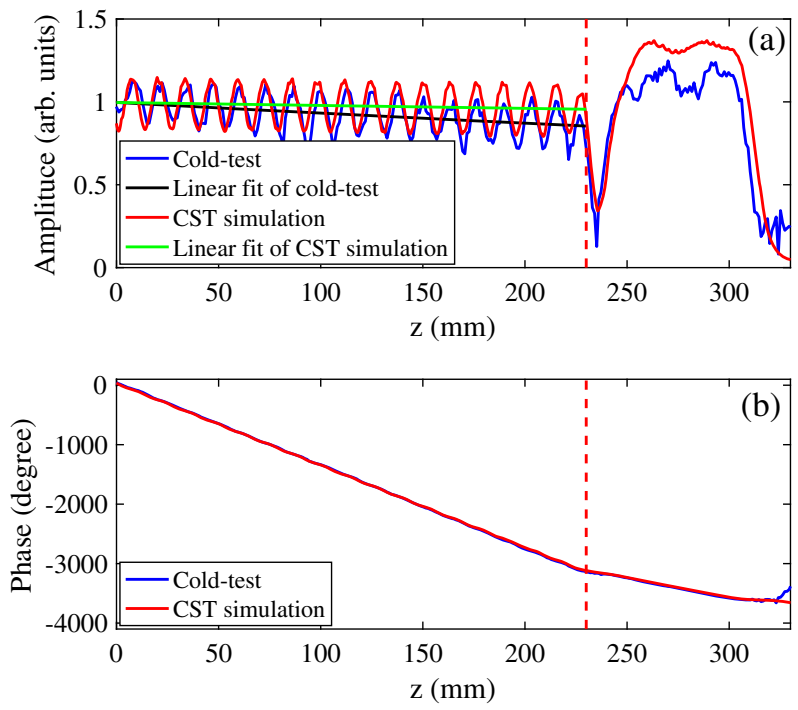

FIG. 11. Normalized amplitude (a) and phase (b) of the on-axis electric field in the dielectric-loaded power extractor at $11.7 \mathrm{GHz}$. Because the mode launcher was inserted into the dielectric tube, the field at the beam entrance could not be measured. The origin of the horizontal axis has been shifted accordingly. Vertical dashed lines indicate the boundary between the uniform section (left) and the tapered section/coupler (right).

The corresponding rf loss of the uniform section is $-0.4 \mathrm{~dB}$. For the black line, the fitted $\alpha$ of $0.67 \mathrm{~m}^{-1}$ indicates the quality factor of the uniform section drops from the nominal number of 3393 to $\sim 950$. The corresponding $\mathrm{rf}$ loss of the uniform section is $-1.5 \mathrm{~dB}$. The difference in the rf loss $(1.5-0.4=1.1 \mathrm{~dB})$ is consistent with the aforementioned value between measurement and simulation $(1.3 \mathrm{~dB})$. The higher structure loss results in a $\sim 12 \%$ reduction of the generated rf power compared to the theoretical calculation and the CST Microwave Studio simulation. According to Eqs. (9) and (13), the higher loss causes no major change to the rf pulse shape.

The phase velocity was calculated from the phase distribution for both the bead-pull measurement and the CST simulation. The phase of the on-axis electric field follows $\phi(z)=\phi_{0}-\beta z$. Therefore, the propagation constant and the phase velocity can be derived as

$$
\left\{\begin{array}{l}
\beta=\frac{d \phi(z)}{d z} \\
v_{p}=\frac{2 \pi f}{\beta}
\end{array},\right.
$$

where $f$ is the driving frequency in the bead-pull measurement.

\section{Synchronization frequency}

The dispersion curve of the structure was obtained by measuring the phase distribution at various frequencies, as illustrated in Fig. 12. The intersection of the dispersion curve (blue) and the line representing the speed of the light 


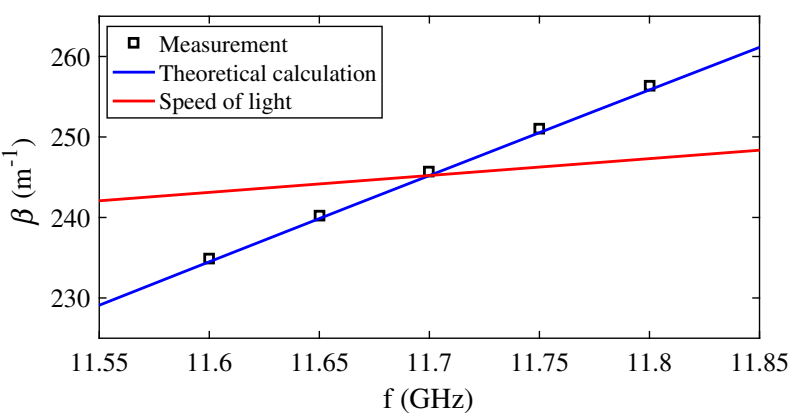

FIG. 12. Dispersion curve of the dielectric-loaded power extractor.

(red) indicates $v_{p}=c$ when the beam is continuously decelerated via Cherenkov radiation and generates wakefield at the corresponding frequency. The measurement results agree well with the theoretical calculation of the uniform section by Eqs. (1) and (4), showing the wakefield frequency is $\sim 11.69 \mathrm{GHz}$, close to the design value of $11.7 \mathrm{GHz}$.

To coherently enhance the wakefield from a multi-bunch train generated by the $1.3 \mathrm{GHz}$ rf linac at AWA, the interval between each bunch should be $1 / 11.69 \times 9=769.9 \mathrm{ps}$, which is $0.7 \mathrm{ps}$ longer than the nominal value. The small offset can be compensated for by launching the bunches in the photocathode $\mathrm{rf}$ gun every $360.3^{\circ}$ instead of every $360^{\circ}$. The accumulated launching phase difference for the 8 -bunch train is $\sim 2^{\circ}$ which does not influence the beam transportation.

\section{HIGH-POWER TEST}

\section{A. Experimental setup}

The experimental setup used at AWA is illustrated in Fig. 13. The input linearly polarized ultraviolet (UV, $248 \mathrm{~nm}$ ) laser for the photocathode gun is produced by a Ti:Sapphire laser system and a third harmonic converter. It has a variable FWHM pulse length of $1.5-10$ ps and an energy up to $10 \mathrm{~mJ}$ amplified by a $\mathrm{KrF}$ excimer [55]. A UV multi-splitter consisting of four beamsplitters and three delay lines [63] is used to generate an 8-bunch laser pulse train with $\lambda$ interval. The length of the three delay lines is $\lambda$, $2 \lambda$, and $4 \lambda$ respectively. The value of $\lambda$ is first coarsely set to the $1.3 \mathrm{GHz}$ wavelength $(230.6 \mathrm{~mm})$ so that the bunches are launched in every rf bucket of the L-band gun. To fine tune the beam launching phase, the laser pulse interval can be remotely adjusted by moving the position of the mirrors mounted on delay lines. Individual pulses and certain pulse combinations can be achieved by the removable laser blockers. The transmission/reflection of the splitters is nominally $50 / 50( \pm 2)$ percent, but this also depends on the polarization angle, which can be adjusted by a laser polarizer before the multi-splitter. After optimization, the charge balance of the 8-bunch train reached 1.00, 0.95, $1.00,0.94,0.91,0.92,0.92$, and 0.85 in this study. The total charge can be adjusted by a laser attenuator mounted at the entrance of the multi-splitter stage.

The electron beam was produced by the AWA's L-band 1.6-cell rf photocathode gun with a cathode gradient of $62 \mathrm{MV} / \mathrm{m}$ and a launching phase of $50^{\circ}[64,65]$. The cesium telluride cathode has a high quantum efficiency of $\sim 10 \%$, which could achieve a maximum charge of $\sim 600 \mathrm{nC}$ [66] per pulse train. The transverse laser spot size was set to $\sim 22 \mathrm{~mm}$ in order to mitigate the space charge effect so that the electron bunch length could stay short. After the rf gun and the six 7-cell $\pi$-mode L-band standing wave accelerating cavities [67], the beam was accelerated to $65 \mathrm{MeV}$, focused by the quadrupoles, and passed through the dielectric-loaded power extractor.

The main diagnostics used during the experiment included two integrating current transformers (ICT) [68] located before and after the structure to measure the charge, as well as an rf pickup antenna [69] installed at the output waveguide to measure the generated rf power. The signals were recorded by a 4-channel oscilloscope with a $50 \mathrm{GS} / \mathrm{s}$ sampling rate and a $20 \mathrm{GHz}$ bandwidth.

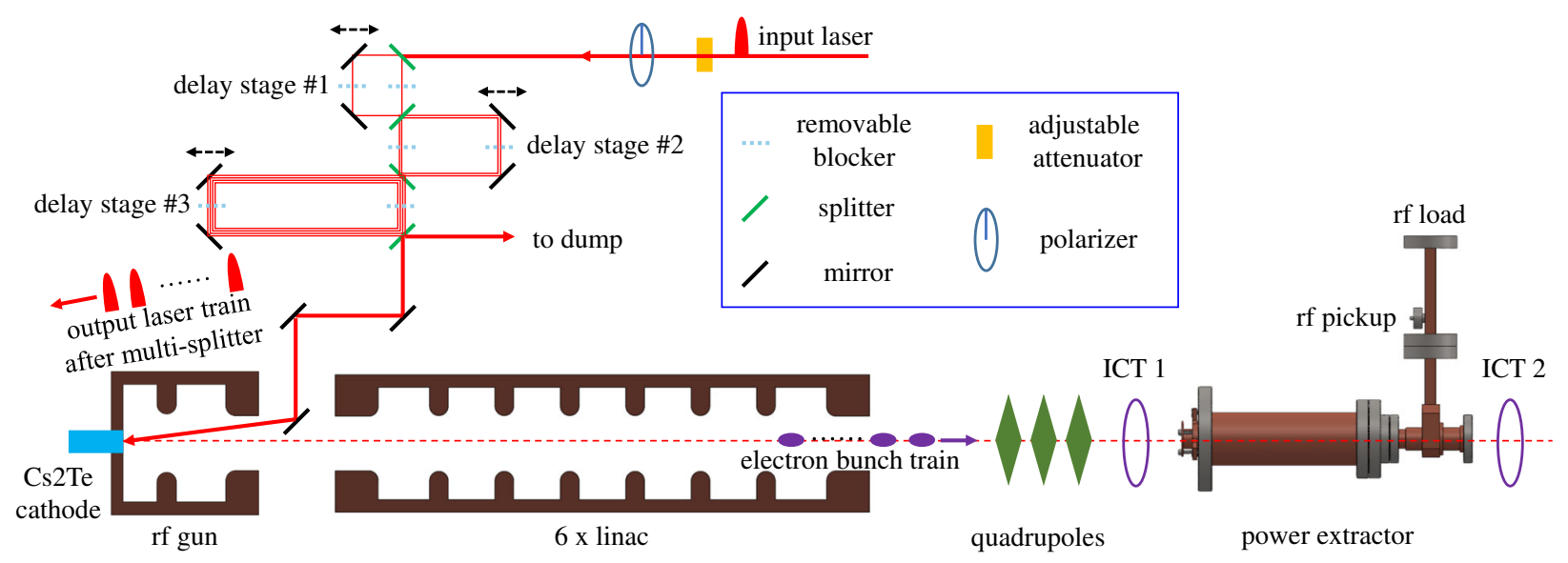

FIG. 13. Schematic of the high-power experiment at AWA. 


\section{B. Experimental results}

\section{Fine tuning of the delay stages}

The launching phases of the bunches were fine-tuned using a low-charge multibunch train by adjusting the delay stages to maximize the generated rf power. Figure 14 shows an example of tuning the first delay stage using the first two bunches. By ignoring the delay of one L-band period and assuming the same charge, the rf field excited by the two bunches can be expressed as $E_{1}(t)=E \cos (\omega t)$ and $E_{2}(t)=E \cos (\omega t+\Delta \theta)$, where $E$ is the field amplitude and $\Delta \theta$ is the relative phase difference. The superposed field amplitude is therefore equal to $E_{12}=E \times 2 \cos (\triangle \theta / 2)$. When moving the delay stage to change $\Delta \theta$, the experimental results agree very well with the CST Particle Studio simulation in which $E_{2}$ is duplicated from $E_{1}$ with a phase shift. Using the same method, the second and the third delay stages can be tuned with the first two 2-bunch trains (bunch Nos.1-2 and bunch Nos.3-4) and the two 4-bunch trains (bunch Nos.
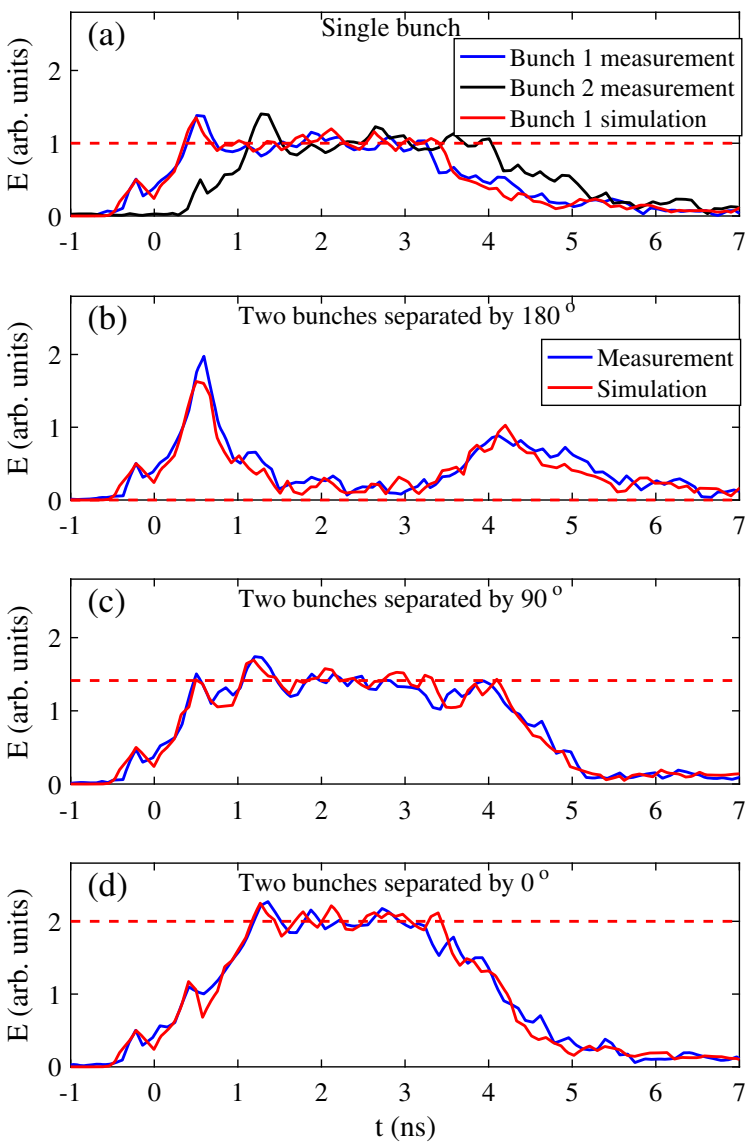

FIG. 14. Comparison between rf pulses measured by the pickup and the output port signal from the CST Particle Studio simulation when tuning the first stage. The same amplitude normalization factor has been applied to all signals to show the relative amplitude change. The red dashed lines in (a-d) denote 1 , $2 \times \cos \left(180^{\circ} / 2\right), 2 \times \cos \left(90^{\circ} / 2\right)$, and $2 \times \cos \left(0^{\circ} / 2\right)$, respectively. The time, $t=0$, is set to be the same as that in Fig. 6 .
1-4 and bunch Nos.5-8), respectively. The accuracy of the tuning was better than $1^{\circ}$ in the L-band or $9^{\circ}$ in the $X$-band.

\section{High-power generation}

We gradually increased the duration and power level of the generated rf pulses by increasing the number and the charge of bunches in the drive train after setting the delay stages. This process is similar to $\mathrm{rf}$ conditioning in externally rf-driven metallic high-gradient structures where the power is increased to raise the gradient [70,71]. The single bunch (bunch No.1), the 2-bunch train (bunch Nos.1-2), the 4-bunch train (bunch Nos.1-4), and the 8-bunch train (bunch Nos.1-8) were applied successively to increase the flat-top duration. With each bunch train configuration, the charge slowly increased from zero to the maximum value $(50 \mathrm{nC}$ for the single bunch, $100 \mathrm{nC}$ for the 2-bunch train, $195 \mathrm{nC}$ for the 4-bunch train, and $360 \mathrm{nC}$ for the 8-bunch train) to improve the power level, as illustrated
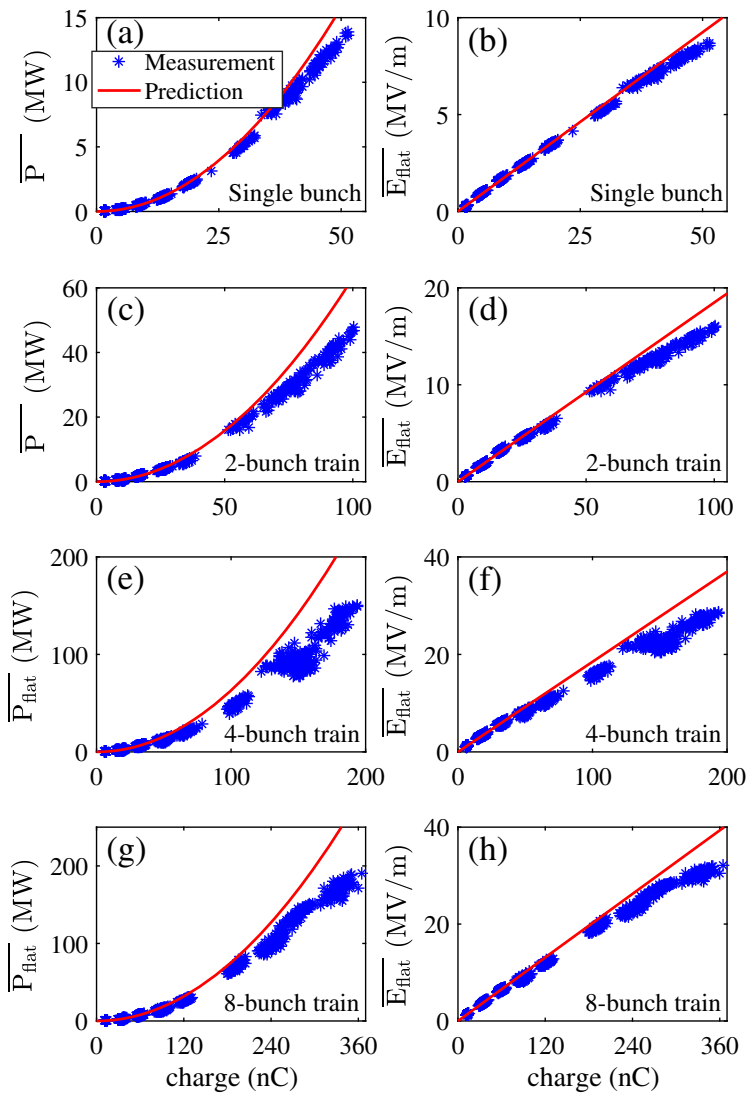

FIG. 15. Comparison of the rf power (left) and the wakefield gradient (right) of the flat-top between the experimental result and the theoretical prediction. In the experimental results, the rf power is the measurement data and the wakefield gradient is the derived value by Eq. (19) based on the uniform section parameters listed in Table I. In the prediction, the form factor is fixed at 0.96; the quality factor of the uniform section is set to 950 according to the cold-test results; and the realistic charge balance is used. 
in Fig. 15. The solenoids along the beamline (not shown in Fig. 13) and the quadrupoles were adjusted to ensure $\sim 100 \%$ charge transmission through the structure.

When driven by the 8-bunch train, with a total charge of $360 \mathrm{nC}$, the structure successfully generated $\sim 200 \mathrm{MW}$, $\sim 3$ ns flat-top rf pulses. The corresponding wakefield gradient derived by Eq. (19) was $\sim 32 \mathrm{MV} / \mathrm{m}$. The maximum $U_{\text {loss }}$ of the bunches was less than $6 \mathrm{MeV}$, according to Eq. (15).

rf breakdown can lead to an abrupt power drop within the transmitted/generated rf pulses [70,71]. However, this was not observed during the experiment. Figure 16 compares the simulated rf pulse shapes and the measured ones with the highest charge. There is good agreement, which indicates that there was no structure damage during the measurement.

It should be noted that transverse modes could be excited when the beam was off-axis and they might lead to inaccurate power measurements. The first and most harmful transverse mode that synchronizes with the beam in the presented structure is the cylindrical $\mathrm{HEM}_{11}$ mode [22] working at $\sim 10.7 \mathrm{GHz}$. In this study, the effect of this mode
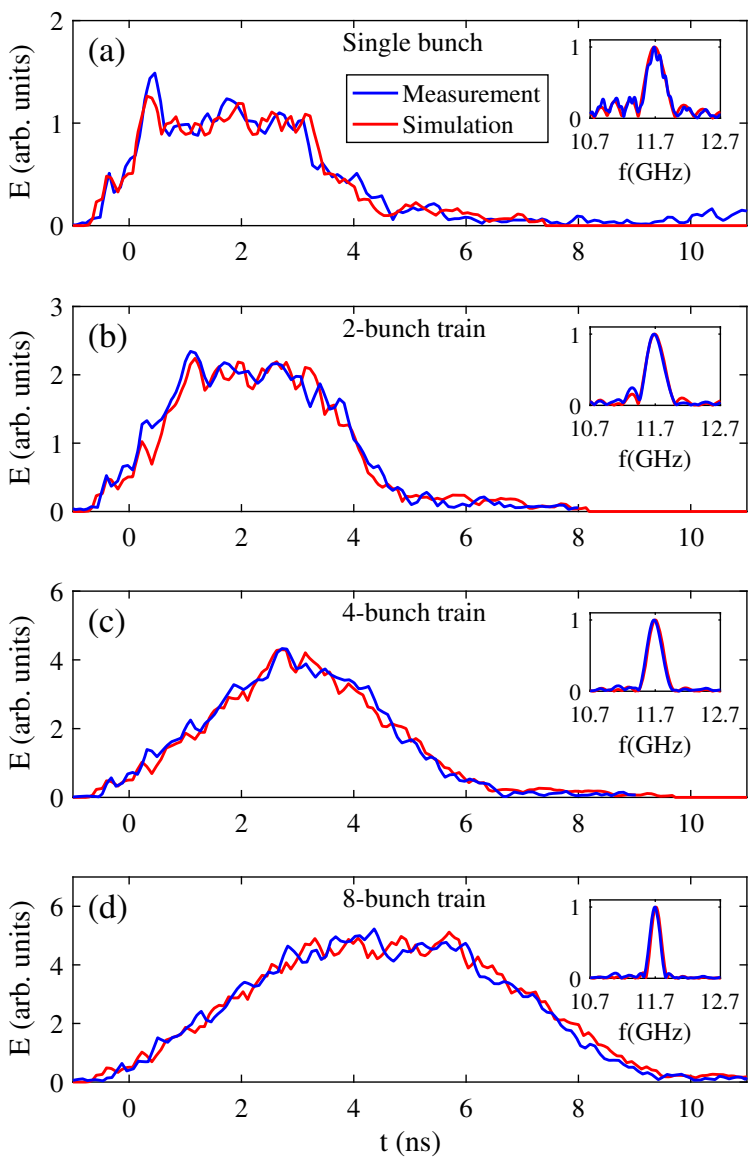

FIG. 16. Comparison of the field envelope and the spectrum (inset) of the coupler output rf signal between the experimental measurement and the CST Particle Studio simulation. The time, $t=0$, is set to be the same as that in Fig. 6 . on the power measurement is negligible due to the following factors: (1) The coupling of the mode to the coupler is weak according to CST Particle Studio simulation, in which the frequency component could not be found in the coupler output spectrum when the beam is offaxis. (2) The beam was maintained near the z-axis in experiment to achieve high transmission. This statement is supported by the absence of the $\sim 10.7 \mathrm{GHz}$ frequency component in the measured spectrum, as illustrated in the inset of Fig. 16.

\section{Data analysis}

Beam dynamics simulations with the general particle tracer (GPT) code [72] were conducted to understand the discrepancy between the measured rf power and the predicted value when pushing the power. The rms bunch length was simulated from the cathode to the power extractor entrance with similar beam conditions and beamline settings as those in the experiment. The space charge as well as the reduced field in the rf gun/linacs due to the beam loading effect [67] were taken into consideration.

\section{Single bunch}

The derived $\overline{E_{\text {flat }}}$ from the single bunch measurement is linearly proportional to the charge in the regime where the charge is less than $20 \mathrm{nC}$, as illustrated in Fig. 15(b). Therefore, the form factor can be fitted to be 0.96 from Eqs. (18) and (9). The corresponding rms bunch length is $1.2 \mathrm{~mm}$, which agrees well with the GPT simulated value of $1.1 \mathrm{~mm}$.

We suspect that the discrepancy of $\overline{E_{\text {flat }}}$ between the measurement and the prediction with increasing charge is caused by a decrease of the form factor. This is a result of a longer bunch length due to space charge forces and beam loading effects. The form factor obtained from a fit of the high-charge data is $\sim 7 \%$ lower, which corresponds to an rms bunch length of $2.0 \mathrm{~mm}$; this is in reasonable agreement with the GPT simulated value of $1.6 \mathrm{~mm}$. The difference might be caused by the non-Gaussian distribution of the drive beam longitudinal profile in the experiment.

\section{Bunch train}

The aforementioned form factor of 0.96 is used to calculate the generated rf power when driven by multibunch trains. The measurement results show good agreement with the predictions with low charges $(\leq 20 \mathrm{nC}$ per bunch), as illustrated in Fig. 15(c-h).

The deviation between the measurement results and the theoretical predictions observed at high charges is similar to the single bunch case. With the highest charge of the 8-bunch train, the measured rf power is $\sim 70 \%$ of the prediction. It is worth mentioning that the percentage is close to that reported with an $X$-band metallic power 
extractor tested at AWA under similar beam conditions [25]. Therefore, the low power generation is more likely caused by factors other than the structure itself. Two possible factors, the bunch lengthening effect at high charges and the error in launching phase, have been discussed as follows.

The rms bunch length of the last bunch is simulated to be $1.9 \mathrm{~mm}$, slightly longer than the first one due to the beam loading effect in the rf gun and linacs. The average bunch length of the 8-bunch train is $\sim 1.7 \mathrm{~mm}$, which leads to a form factor of 0.91 and a power reduction of $10 \%$.

The worst-case scenario of the stage tuning would be that all three delay stages were off by $9^{\circ}$ in the $X$-band to the same direction, causing the launching phase (in the $X$ band) of the eight bunches to be $0^{\circ}, 9^{\circ}, 9^{\circ}, 18^{\circ}, 9^{\circ}, 18^{\circ}, 18^{\circ}$, and $27^{\circ}$, respectively. Because the flat-top can be reached by five bunches, the superposed wakefield amplitude when ignoring the structure loss will be $\cos \left(0^{\circ}\right)+\cos \left(9^{\circ}\right)+$ $\cos \left(9^{\circ}\right)+\cos \left(18^{\circ}\right)+\cos \left(9^{\circ}\right)=4$.9. This results in an $\sim 2 \%$ reduction in the field amplitude and $\sim 3 \%$ reduction in the corresponding power compared to the ideal case.

Therefore, these two factors could lead to a maximum of $\sim 13 \%$ lower generated power at high charges. The remaining difference between the analysis and the experimental result might be caused by non-Gaussian longitudinal distribution, multipacting of the rf pickup [69], and other unknown factors. A detailed study of the time structure of the drive bunches was not performed in this experiment, but will be investigated in the future.

\section{FUTURE STUDY}

In this section, we present the design of a study of a future dielectric-loaded power extractor in order to maximize the generated rf power beyond the 200 MW achieved in this study. This is done by optimizing the design of the dielectric-loaded power extractor while assuming similar beam parameters to those achieved in this study. The drive beam is assumed to contain an 8-bunch train with $\sigma_{z}$ of $1.2 \mathrm{~mm}, T_{b}$ of $769.2 \mathrm{ps}, Q_{b}$ of $50 \mathrm{nC}$, and perfect charge balance. This represents a total drive train charge of $400 \mathrm{nC}$, which is below the $600 \mathrm{nC}$ previously demonstrated at AWA [66]. $L_{s t}$ and $v_{g}$ are fixed at $26 \mathrm{~cm}$ and $0.184 \mathrm{c}$, respectively so that $N_{\text {rise }}=4$ and $t_{\text {flat }}=3.1 \mathrm{~ns}$. The frequency choice of the future structure is considered for L-band harmonics starting from the C-band $(7.8 \mathrm{GHz})$ to the Ka-band $(32.5 \mathrm{GHz})$. In addition, several inner radii are used for each structure design. For each combination of $\omega_{0}, v_{g}$, and $a, \epsilon_{r}$ is calculated from Eqs. (1) and (4), with the upper limit set to 20 for the ease to obtain low-loss dielectric materials.

The optimization results of the uniform section are shown in Fig. 17. The maximum achievable rf power increases when reducing the inner radius, and the corresponding operation frequency shifts to higher values. When $a \leq 4 \mathrm{~mm}$, a gigawatt level can be obtained around
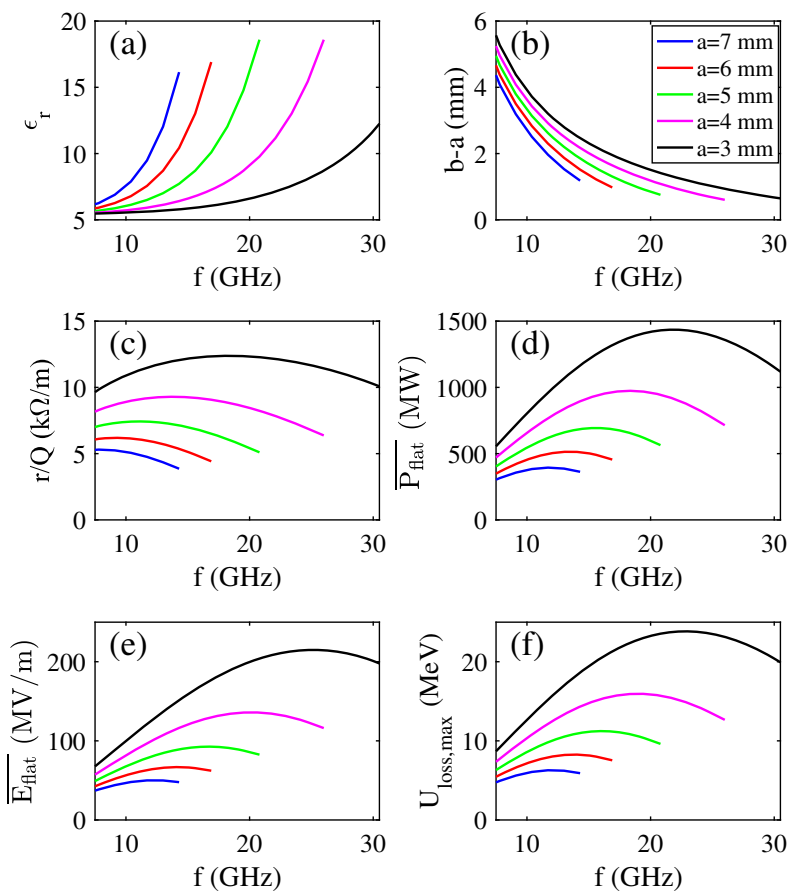

FIG. 17. EM and wakefield properties of the uniform section as a function of the operation frequency with various inner radii. (a) Dielectric constant. (b) Dielectric thickness. (c) $r / Q$. (d) $\overline{P_{\text {flat }}}$. (e) $\overline{E_{\text {flat }}}$. (f) Maximum $U_{\text {loss }}$.

$20 \mathrm{GHz}$. In this regime, the dielectric-loaded power extractors are feasible in fabrication: the required low-loss dielectric materials, such as Forsterite $\left(\epsilon_{r}=6.64\right)$ [22] and alumina, are commercially available, and the wall thickness is $\sim 1.5 \mathrm{~mm}$. The structures are also physically achievable: the corresponding gradient is well below the $\mathrm{GV} / \mathrm{m}$ level

TABLE II. Parameters of the uniform section of a future gigawatt dielectric-loaded power extractor using AWA drive beam conditions.

\begin{tabular}{lc}
\hline \hline Parameter & Value \\
\hline Dielectric material & Forsterite \\
Dielectric constant & 6.64 \\
Loss tangent & $1 \times 10^{-4}$ \\
Frequency & $19.5 \mathrm{GHz}(15 \times 1.3 \mathrm{GHz})$ \\
Inner radius & $3.1 \mathrm{~mm}$ \\
Outer radius & $4.6 \mathrm{~mm}$ \\
Length & $26 \mathrm{~cm}$ \\
Outer boundary material & Copper \\
Outer boundary conductivity & $5.8 \times 10^{7} \mathrm{~S} / \mathrm{m}$ \\
Quality factor & 3177 \\
$r / Q$ & $11.93 \mathrm{k} \Omega / \mathrm{m}$ \\
Group velocity & $0.184 \mathrm{c}$ \\
$E_{\text {max,surface }} / E_{\text {axis }}$ & 1 \\
Flat-top duration & $3.1 \mathrm{~ns}$ \\
$P_{\text {flat }}$ & $1.36 \mathrm{GW}$ \\
$E_{\text {flat }}$ & $190 \mathrm{MV} / \mathrm{m}$ \\
Maximum $U_{\text {loss }}$ & $22.2 \mathrm{MeV}$ \\
\hline \hline
\end{tabular}


reported in previous rf breakdown tests [44] and CWA experiments [20], and the maximum $U_{\text {loss }}$ of the bunches is $\sim 1 / 3$ of the AWA beam energy. The detailed parameters of a possible design are provided in Table II.

The major challenge in using this future structure will be transmitting the high-charge drive beam through the structure. Maintaining high transmission requires several efforts: improve the beam quality via low emittance (the normalized emittance in the high-charge case was simulated to be $128 \mu \mathrm{m}$, but is expected to be much higher in the current experiment due to the laser profile and the asymmetric rf gun [65], which will be improved soon) and short bunch length [73], implement beam breakup control [74], add HOM damping features [61], and other tasks.

Other challenges include designing rf components ( $\mathrm{rf}$ coupler, matching section, load, and direction coupler) for gigawatt power level, studying rf breakdown and multipacting for HOM-damped structures, and characterizing the time structure of the bunch train.

The next step beyond generating the gigawatt power short rf pulse will be to increase the pulse length up to $20 \mathrm{~ns}$ as in the AFLC baseline design. The number of drive bunches in a train needs to be a power of 2 , based on the current bunch-train generation method at AWA. Therefore, the minimal bunch number would be 32 , according to Eq. (16); this could be achieved by adding two more beamsplitters to the current laser setup. The generation of $1 \mathrm{GW}, 20 \mathrm{~ns}$ rf pulses at AWA is possible because the rf pulse energy $(20 \mathrm{~J})$ is lower than the maximum available beam energy ( $39 \mathrm{~J}$, calculated from a $600 \mathrm{nC}, 65 \mathrm{MeV}$ drive beam). The structure will be optimized in a future study.

\section{SUMMARY}

The DLS is a promising candidate, as both the power extractor and the accelerator, for short-pulse two-beam acceleration, because it has the advantages of simple geometry, low fabrication cost, high group velocity with reasonable shunt impedance, and potential to withstand $\mathrm{GV} / \mathrm{m}$ gradients. In order to demonstrate rf power generation beyond $100 \mathrm{MW}$, an $X$-band $11.7 \mathrm{GHz}$ dielectric-loaded power extractor was developed and tested at AWA. In this article, we present a detailed development process, including design, simulation, fabrication, cold test, and highpower test. Driven by 8-bunch trains with a total charge of $360 \mathrm{nC}$, the structure successfully generated $\sim 200 \mathrm{MW}$, $\sim 3$ ns flat-top rf pulses without rf breakdown. Plans for future structure optimization show that gigawatt power levels are feasible at AWA by using higher-frequency structures with smaller apertures. This work benefits the research of power extractors in the SWFA scheme.

\section{ACKNOWLEDGMENTS}

The work at AWA is funded through the U.S. Department of Energy (DOE) Office of Science under
Contract No. DE-AC02-06CH11357. The work by Euclid Techlabs, LLC, is funded through the U.S. DOE under Small Business Innovation Research Contract No. DESC00011299. We would like to thank Cho $\mathrm{Ng}$, Zenghai Li, Liling Xiao, and Lixin Ge at SLAC for the ACE3P simulation. We would also like to thank Kiran Kumar Kovi and Gongxiaohui Chen from Euclid Techlabs, LLC, as well as Christopher Marshall and Michael Schwartz from the Chemical Sciences and Engineering Division at Argonne National Laboratory for the metallic coating of the dielectric tube.

\section{APPENDIX: POWER LOSS OF A METALLIC WALL WITH A THIN COATING}

In this Appendix, we derive the rf power loss of a metallic wall with a thin coating in the structure. The calculation model is illustrated in Fig. 18, where regions 1, 2 , and 3 represent the dielectric material with dielectric constant of $\epsilon_{1}$, the thin metallic coating with conductivity of $\sigma_{2}$, and the bulk metallic wall with conductivity of $\sigma_{3}$, respectively.

Derivation assumptions in this section are as follows: (1) The rf loss in the dielectric material near the surface is ignored because it is significantly lower than that in the metallic materials. (2) Only a plane wave with $E_{x}$ and $H_{y}$ components propagating in $\mathrm{z}$ direction is considered.

In the following derivation, the subscripts " $1,2,3$ " denote the corresponding region; the plus and minus superscripts denote the forward wave moving in the $+z$ direction and the backward wave moving in the $-z$ direction, respectively.

The field in region 1 can be expressed as

$$
\left\{\begin{aligned}
E_{1}(z) & =E_{1}^{+} e^{-j \beta_{1} z}+E_{1}^{-} e^{+j \beta_{1} z} \\
& =\eta_{1} H_{1}^{+} e^{-j \beta_{1} z}-\eta_{1} H_{1}^{-} e^{+j \beta_{1} z} \\
H_{1}(z) & =H_{1}^{+} e^{-j \beta_{1} z}+H_{1}^{-} e^{+j \beta_{1} z}
\end{aligned}\right.
$$

where $\beta_{1}$ is the propagation constant; $\eta_{1}=\sqrt{\mu /\left(\epsilon_{0} \epsilon_{1}\right)}$ is the wave impedance; and $E_{1}^{+,-}$and $H_{1}^{+,-}$are the field amplitudes.

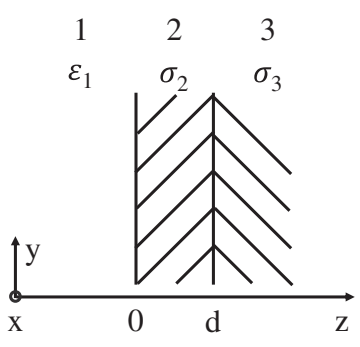

FIG. 18. Calculation model of the rf loss of a metallic wall with a thin coating. 
The field in region 2 can be expressed as

$$
\left\{\begin{aligned}
E_{2}(z) & =E_{2}^{+} e^{-j k_{2} z}+E_{2}^{-} e^{+j k_{2} z} \\
& =\eta_{2} H_{2}^{+} e^{-j k_{2} z}-\eta_{2} H_{2}^{-} e^{+j k_{2} z}, \\
H_{2}(z) & =H_{2}^{+} e^{-j k_{2} z}+H_{2}^{-} e^{+j k_{2} z}
\end{aligned}\right.
$$

where $k_{2}=(1-j) \sqrt{\omega_{0} \mu \sigma_{2} / 2}$ is the propagation constant; $\eta_{2}=(1+j) \sqrt{\omega_{0} \mu /\left(2 \sigma_{2}\right)}$ is the wave impedance; and $E_{2}^{+,-}$and $H_{2}^{+,-}$are the field amplitudes.

The field in region 3 can be expressed as

$$
\left\{\begin{aligned}
E_{3}(z) & =E_{3}^{+} e^{-j k_{3} z} \\
& =\eta_{3} H_{3}^{+} e^{-j k_{3} z} \\
H_{3}(z) & =H_{3}^{+} e^{-j k_{3} z}
\end{aligned}\right.
$$

where $k_{3}=(1-j) \sqrt{\omega_{0} \mu \sigma_{3} / 2}$ is the propagation constant; $\eta_{3}=(1+j) \sqrt{\omega_{0} \mu /\left(2 \sigma_{3}\right)}$ is the wave impedance; and $E_{3}^{+}$ and $\mathrm{H}_{3}^{+}$are the field amplitudes.

The boundary conditions are given by

$$
\left\{\begin{array}{l}
\left.E_{1}\right|_{z=0}=\left.E_{2}\right|_{z=0} \\
\left.H_{1}\right|_{z=0}=\left.H_{2}\right|_{z=0} \equiv H_{t} \\
\left.E_{2}\right|_{z=d}=\left.E_{3}\right|_{z=d} \\
\left.H_{2}\right|_{z=d}=\left.H_{3}\right|_{z=d}
\end{array},\right.
$$

where $d$ is the thickness of the coating.
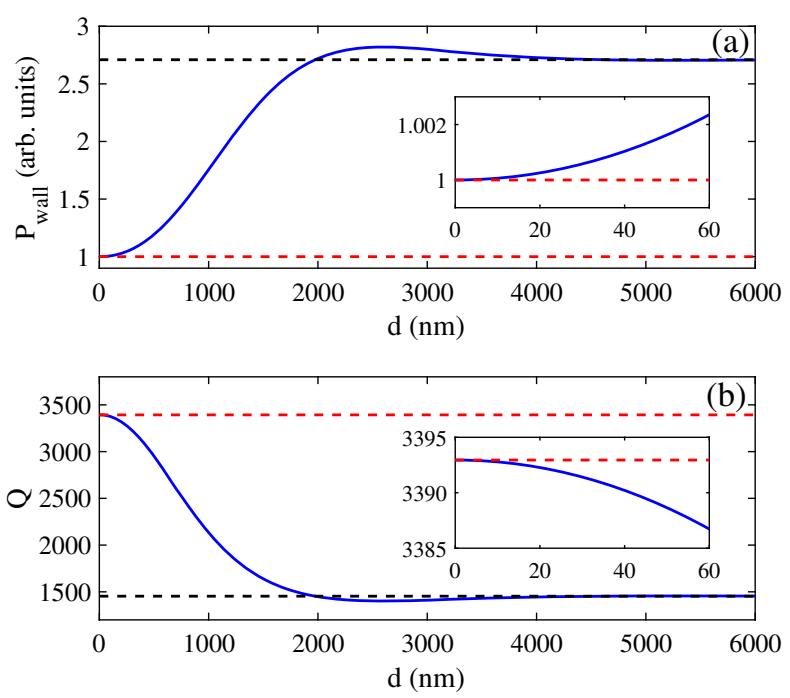

FIG. 19. Metallic wall loss (a, normalized to that of copper without coating) and quality factor (b) of the uniform section in the presented dielectric-loaded power extractor as a function of the chromium coating thickness (solid blue lines). The values using bulk copper ( $d=0$, red dashed lines) and bulk chromium ( $d=+\infty$, black dashed lines) are also plotted for comparison. Inset: magnified view at small coating thickness.
By solving Eqs. (A1)-(A4), the loss per unit area of the metallic wall with the coating can be calculated as

$$
\begin{aligned}
P_{\text {wall }} & =\operatorname{Re}\left(\frac{1}{2} E_{1}^{+} \times H_{1}^{+}\right)-\operatorname{Re}\left(\frac{1}{2} E_{1}^{-} \times H_{1}^{-}\right) \\
& =\frac{1}{2} \eta_{1}\left(\left|\frac{1}{1+K}\right|^{2}-\left|\frac{K}{1+K}\right|^{2}\right)\left|H_{t}\right|^{2},
\end{aligned}
$$

where the constant $K$ is defined as

$$
K=\frac{\left(\eta_{1}-\eta_{2}\right)\left(\eta_{2}+\eta_{3}\right)+\left(\eta_{1}+\eta_{2}\right)\left(\eta_{2}-\eta_{3}\right) e^{-2 j k_{2} d}}{\left(\eta_{1}+\eta_{2}\right)\left(\eta_{2}+\eta_{3}\right)+\left(\eta_{1}-\eta_{2}\right)\left(\eta_{2}-\eta_{3}\right) e^{-2 j k_{2} d}} .
$$

In the presented dielectric-loaded power extractor, the wall loss of bulk copper with a chromium layer can be therefore calculated by replacing $H_{t}$ with $\left.H_{\theta, 2}\right|_{\rho=b}$ in Eq. (A5). The wall loss and the corresponding quality factor of the uniform section are illustrated in Fig. 19, which indicates that the reduction of the quality factor is negligible when the chromium layer is only $10 \mathrm{~nm}$ thick.

[1] E. R. Colby and L. K. Len, Roadmap to the future, Rev. Accel. Sci. Techol. 09, 1 (2016).

[2] L. K. Len, in Report of the DOE Advanced Accelerator Concepts Research Roadmap Workshop, Gaithersburg, US (DOE, Gaithersburg, MD, 2016).

[3] B. Cros and P. Muggli, in Report on the Advanced and Novel Accelerators for High Energy Physics Roadmap Workshop, CERN, Geneva (CERN, Geneva, Switzerland, 2017).

[4] E. Esarey, C. Schroeder, and W. Leemans, Physics of laserdriven plasma-based electron accelerators, Rev. Mod. Phys. 81, 1229 (2009).

[5] W. Leemans, A. Gonsalves, H.-S. Mao, K. Nakamura, C. Benedetti, C. Schroeder, C. Tóth, J. Daniels, D. Mittelberger, S. Bulanov et al., Multi-GeV Electron Beams from Capillary-Discharge-Guided Subpetawatt Laser Pulses in the Self-Trapping Regime, Phys. Rev. Lett. 113, 245002 (2014).

[6] S. Steinke, J. Van Tilborg, C. Benedetti, C. Geddes, C. Schroeder, J. Daniels, K. Swanson, A. Gonsalves, K. Nakamura, N. Matlis et al., Multistage coupling of independent laser-plasma accelerators, Nature (London) 530, 190 (2016).

[7] E. Peralta, K. Soong, R. England, E. Colby, Z. Wu, B. Montazeri, C. McGuinness, J. McNeur, K. Leedle, D. Walz et al., Demonstration of electron acceleration in a laserdriven dielectric microstructure, Nature (London) 503, 91 (2013).

[8] R. J. England, R. J. Noble, K. Bane, D. H. Dowell, C.-K. Ng, J. E. Spencer, S. Tantawi, Z. Wu, R. L. Byer, E. Peralta et al., Dielectric laser accelerators, Rev. Mod. Phys. 86, 1337 (2014). 
[9] K. P. Wootton, Z. Wu, B. M. Cowan, A. Hanuka, I. V. Makasyuk, E. A. Peralta, K. Soong, R. L. Byer, and R. J. England, Demonstration of acceleration of relativistic electrons at a dielectric microstructure using femtosecond laser pulses, Opt. Lett. 41, 2696 (2016).

[10] M. Hogan, C. Barnes, C. Clayton, F. Decker, S. Deng, P. Emma, C. Huang, R. Iverson, D. Johnson, C. Joshi et al., Multi-GeV Energy Gain in a Plasma-Wakefield Accelerator, Phys. Rev. Lett. 95, 054802 (2005).

[11] M. Litos, E. Adli, W. An, C. Clarke, C. Clayton, S. Corde, J. Delahaye, R. England, A. Fisher, J. Frederico et al., High-efficiency acceleration of an electron beam in a plasma wakefield accelerator, Nature (London) 515, 92 (2014).

[12] S. Corde, E. Adli, J. Allen, W. An, C. Clarke, C. Clayton, J. Delahaye, J. Frederico, S. Gessner, S. Green et al., Multigigaelectronvolt acceleration of positrons in a self-loaded plasma wakefield, Nature (London) 524, 442 (2015).

[13] W. Gai, P. Schoessow, B. Cole, R. Konecny, J. Norem, J. Rosenzweig, and J. Simpson, Experimental Demonstration of Wake-Field Effects in Dielectric Structures, Phys. Rev. Lett. 61, 2756 (1988).

[14] C. Jing, A. Kanareykin, J. Power, M. Conde, Z. Yusof, and W. Gai, Observation of enhanced transformer ratio in collinear wakefield acceleration, AIP Conf. Proc. 877, 511 (2006).

[15] F. Gao, M. Conde, W. Gai, C. Jing, R. Konecny, W. Liu, J. Power, T. Wong, and Z. Yusof, Design and testing of a $7.8 \mathrm{GHz}$ power extractor using a cylindrical dielectricloaded waveguide, Phys. Rev. Accel. Beams 11, 041301 (2008).

[16] C. Jing, A. Kanareykin, J. Power, M. Conde, W. Liu, S. Antipov, P. Schoessow, and W. Gai, Experimental Demonstration of Wakefield Acceleration in a Tunable Dielectric Loaded Accelerating Structure, Phys. Rev. Lett. 106, 164802 (2011).

[17] G. Andonian, D. Stratakis, M. Babzien, S. Barber, M. Fedurin, E. Hemsing, K. Kusche, P. Muggli, B. OShea, X. Wei et al., Dielectric Wakefield Acceleration of a Relativistic Electron Beam in a Slab-Symmetric Dielectric Lined Waveguide, Phys. Rev. Lett. 108, 244801 (2012).

[18] S. Antipov, C. Jing, A. Kanareykin, J. Butler, V. Yakimenko, M. Fedurin, K. Kusche, and W. Gai, Experimental demonstration of wakefield effects in a THz planar diamond accelerating structure, Appl. Phys. Lett. 100, 132910 (2012).

[19] W. Gai, J. G. Power, and C. Jing, Short-pulse dielectric two-beam acceleration, J. Plasma Phys. 78, 339 (2012).

[20] B. OShea, G. Andonian, S. Barber, K. Fitzmorris, S. Hakimi, J. Harrison, P. Hoang, M. Hogan, B. Naranjo, O. Williams et al., Observation of acceleration and deceleration in gigaelectron-volt-per-metre gradient dielectric wakefield accelerators, Nat. Commun. 7, 12763 (2016).

[21] D. Wang, S. Antipov, C. Jing, J. Power, M. Conde, E. Wisniewski, W. Liu, J. Qiu, G. Ha, V. Dolgashev et al., Interaction of an Ultrarelativistic Electron Bunch Train with a W -Band Accelerating Structure: High Power and High Gradient, Phys. Rev. Lett. 116, 054801 (2016).
[22] C. Jing, Dielectric wakefield accelerators, Rev. Accel. Sci. Techol. 09, 127 (2016).

[23] Q. Gao, G. Ha, C. Jing, S. Antipov, J. Power, M. Conde, W. Gai, H. Chen, J. Shi, E. Wisniewski et al., Observation of High Transformer Ratio of Shaped Bunch Generated by an Emittance-Exchange Beam Line, Phys. Rev. Lett. 120, 114801 (2018).

[24] K. Lekomtsev, A. Aryshev, A. Tishchenko, M. Shevelev, A. Lyapin, S. Boogert, P. Karataev, N. Terunuma, and J. Urakawa, Driver-witness electron beam acceleration in dielectric mm-scale capillaries, Phys. Rev. Accel. Beams 21, 051301 (2018).

[25] C. Jing, S. Antipov, M. Conde, W. Gai, G. Ha, W. Liu, N. Neveu, J. Power, J. Qiu, J. Shi et al., Electron acceleration through two successive electron beam driven wakefield acceleration stages, Nucl. Instrum. Methods Phys. Res., Sect. A 898, 72 (2018).

[26] J. Shao, C. Jing, J. Power, M. Conde, and D. Doran, in Proceedings of the 9th International Particle Accelerator Conference, Vancouver, BC, Canada (JACoW, Geneva, Switzerland, 2018), pp. 1539-1541.

[27] X. Lu, M. A. Shapiro, I. Mastovsky, R. J. Temkin, M. Conde, J. G. Power, J. Shao, E. E. Wisniewski, and C. Jing, Generation of High-Power, Reversed-Cherenkov Wakefield Radiation in a Metamaterial Structure, Phys. Rev. Lett. 122, 014801 (2019).

[28] CLIC Conceptual Design Report, Technical Report No. CERN-2012-007.

[29] A. Grudiev, S. Calatroni, and W. Wuensch, New local field quantity describing the high gradient limit of accelerating structures, Phys. Rev. Accel. Beams 12, 102001 (2009).

[30] M. Dal Forno, V. Dolgashev, G. Bowden, C. Clarke, M. Hogan, D. McCormick, A. Novokhatski, B. OShea, B. Spataro, S. Weathersby et al., rf breakdown measurements in electron beam driven $200 \mathrm{GHz}$ copper and copper-silver accelerating structures, Phys. Rev. Accel. Beams 19, 111301 (2016).

[31] H. Zha and A. Grudiev, Design of the Compact Linear Collider main linac accelerating structure made from two halves, Phys. Rev. Accel. Beams 20, 042001 (2017).

[32] X. Lu, M. A. Shapiro, and R. J. Temkin, Modeling of the interaction of a volumetric metallic metamaterial structure with a relativistic electron beam, Phys. Rev. Accel. Beams 18, 081303 (2015).

[33] J. Hummelt, X. Lu, H. Xu, I. Mastovsky, M. Shapiro, and R. Temkin, Coherent Cherenkov-Cyclotron Radiation Excited by an Electron Beam in a Metamaterial Waveguide, Phys. Rev. Lett. 117, 237701 (2016).

[34] E. I. Smirnova, A. S. Kesar, I. Mastovsky, M. A. Shapiro, and R. J. Temkin, Demonstration of a 17-GHz, HighGradient Accelerator with a Photonic-Band-Gap Structure, Phys. Rev. Lett. 95, 074801 (2005).

[35] E. I. Smirnova, I. Mastovsky, M. A. Shapiro, R. J. Temkin, L. M. Earley, and R. L. Edwards, Fabrication and cold test of photonic band gap resonators and accelerator structures, Phys. Rev. Accel. Beams 8, 091302 (2005).

[36] E. I. Simakov, S. A. Arsenyev, C. E. Buechler, R. L. Edwards, W. P. Romero, M. Conde, G. Ha, J. G. Power, E. E. Wisniewski, and C. Jing, Observation of Wakefield 
Suppression in a Photonic-Band-Gap Accelerator Structure, Phys. Rev. Lett. 116, 064801 (2016).

[37] P. Zou, L. Xiao, X. Sun, W. Gai, and T. Wong, Hybrid dielectric and iris-loaded periodic accelerating structure, J. Appl. Phys. 90, 2017 (2001).

[38] C. Jing, A. Kanareykin, S. Kazakov, W. Liu, E. Nenasheva, P. Schoessow, and W. Gai, Development of a dual-layered dielectric-loaded accelerating structure, Nucl. Instrum. Methods Phys. Res., Sect. A 594, 132 (2008).

[39] D. Satoh, M. Yoshida, and N. Hayashizaki, Dielectric assist accelerating structure, Phys. Rev. Accel. Beams 19, 011302 (2016).

[40] A. V. Smirnov and E. Savin, Hybrid metal-dielectric, slow wave structure with magnetic coupling and compensation, Nucl. Instrum. Methods Phys. Res., Sect. A 820, 48 (2016).

[41] A. Cahill, J. Rosenzweig, V. Dolgashev, S. Tantawi, and S. Weathersby, High gradient experiments with $\mathrm{X}$-band cryogenic copper accelerating cavities, Phys. Rev. Accel. Beams 21, 102002 (2018).

[42] P. Hoang, G. Andonian, I. Gadjev, B. Naranjo, Y. Sakai, N. Sudar, O. Williams, M. Fedurin, K. Kusche, C. Swinson et al., Experimental Characterization of Electron-BeamDriven Wakefield Modes in a Dielectric-Woodpile Cartesian Symmetric Structure, Phys. Rev. Lett. 120, 164801 (2018).

[43] Y. Yu, K. Lai, J. Shao, J. Power, M. Conde, W. Liu, S. Doran, C. Jing, E. Wisniewski, and G. Shvets, Transition Radiation in Photonic Topological Crystals: Quasiresonant Excitation of Robust Edge States by a Moving Charge, Phys. Rev. Lett. 123, 057402 (2019).

[44] M. Thompson, H. Badakov, A. Cook, J. Rosenzweig, R. Tikhoplav, G. Travish, I. Blumenfeld, M. Hogan, R. Ischebeck, N. Kirby et al., Breakdown Limits on Gigavolt-per-Meter Electron-Beam-Driven Wakefields in Dielectric Structures, Phys. Rev. Lett. 100, 214801 (2008).

[45] G. Bruck and E. Wicher, Slow transverse magnetic waves in cylindrical guides, J. Appl. Phys. 18, 766 (1947).

[46] R.S. Harvie, A proposed new form of dielectric-loaded wave-guide for linear electron accelerators, Nature (London) 162, 890 (1948).

[47] D. Wang, X. Su, Y. Du, Q. Tian, Y. Liang, L. Niu, W. Huang, W. Gai, L. Yan, C. Tang et al., Non-perturbing THz generation at the Tsinghua University Accelerator Laboratory $31 \mathrm{MeV}$ electron beamline, Rev. Sci. Instrum. 89, 093301 (2018).

[48] E. Chojnacki, W. Gai, C. Ho, R. Konecny, S. Mtingwa, J. Norem, M. Rosing, P. Schoessow, and J. Simpson, Measurement of deflection-mode damping in an accelerating structure, J. Appl. Phys. 69, 6257 (1991).

[49] C. Jing, C. Chang, S. Gold, R. Konecny, S. Antipov, P. Schoessow, A. Kanareykin, and W. Gai, Observation of multipactor suppression in a dielectric-loaded accelerating structure using an applied axial magnetic field, Appl. Phys. Lett. 103, 213503 (2013).

[50] C. Jing, S. Gold, R. Fischer, and W. Gai, Complete multipactor suppression in an X-band dielectric-loaded accelerating structure, Appl. Phys. Lett. 108, 193501 (2016).
[51] J. Shao, S. Antipov, M. Conde, W. Gai, Q. Gao, G. Ha, C. Jing, W. Liu, N. Neveu, J. Power et al., in Proceedings of the 8th International Particle Accelerator Conference, Copenhagen, Denmark (JACoW, Geneva, Switzerland, 2017), pp. 3305-3307.

[52] C. Jing, S. Antipov, A. Kanareykin, and R. Kostin, in Proceedings of the 9th International Particle Accelerator Conference, Vancouver, BC, Canada (JACoW, Geneva, Switzerland, 2018), pp. 464-466.

[53] A. Kanareykin, W. Gai, J. G. Power, and P. Schoessow, in Proceedings of the 11th Advance Accelerator Concepts Workshop, Stony Brook, US (AIP, Melville, NY, 2004), pp. 272-280.

[54] D. Wang, Research on the terahertz wakefield radiation based on the electron bunch train, PhD thesis, Tsinghua University, 2016.

[55] M. Conde, G. Ha, E. Wisniewski, W. Liu, N. Neveu, C. Whiteford, J. Qiu, J. Shao, S. Antipov, L. Zheng et al., in Proceedings of the 8th International Particle Accelerator Conference, Copenhagen, Denmark (JACoW, Geneva, Switzerland, 2017), pp. 2885-2887.

[56] C. Nantista, S. Tantawi, and V. Dolgashev, Low-field accelerator structure couplers and design techniques, Phys. Rev. Accel. Beams 7, 072001 (2004).

[57] V. A. Dolgashev, S. Tantawi, Y. Higashi, T. Higo, and C. Nantista, in Proceedings of the 21st Particle Accelerator Conference, Knoxville, TN, 2005 (IEEE, Piscataway, NJ, 2005), pp. 595-599.

[58] CST Studio, CST, Bad Nauheimer Str. 19, 64289 Darmstadt, Germany.

[59] K. Ko, A. Candel, L. Ge, A. Kabel, R. Lee, Z. Li, C. Ng, V. Rawat, G. Schussman, and L. Xiao, in Proceedings of the 25th International Linear Accelerator Conference, LINAC-2010, Tsukuba, Japan (KEK, Tsukuba, Japan, 2010), pp. 1028-1032.

[60] A. A. Grigoreva, A. V. Tyukhtin, V. V. Vorobev, S. N. Galyamin, and S. Antipov, Radiation of a charge flying in a partially loaded dielectric section of a waveguide, Phys. Rev. Accel. Beams 21, 031302 (2018).

[61] C. Jing, J. Lewandowski, S. Tantawi, A. Kanareykin, P. Schoessow, M. Conde, W. Gai, I. Syratchev, J. Power, V. Dolgashev et al., in Proceedings of the 4th International Particle Accelerator Conference, Shanghai, China (JACoW, Geneva, Switzerland, 2013), pp. 2908-2910.

[62] J. Shi, A. Olyunin, W. Wuensch, and A. Grudiev, in Proceedings of the 25th International Linear Accelerator Conference, LINAC-2010, Tsukuba, Japan (KEK, Tsukuba, Japan, 2010), pp. 97-99.

[63] N. Neveu, G. Ha, E. Wisniewski, W. Liu, C. Whiteford, S. Antipov, M. Conde, C. Jing, W. Gai, D. Doran et al., in Proceedings of the 7th International Particle Accelerator Conference, Busan, Korea (JACoW, Geneva, Switzerland, 2016), pp. 1629-1631.

[64] W. Gai, X. Li, M. Conde, J. Power, and P. Schoessow, A high-charge and short-pulse RF photocathode gun for wake-field acceleration, Nucl. Instrum. Methods Phys. Res., Sect. A 410, 431 (1998).

[65] L. Zheng, J. Shao, Y. Du, J. G. Power, E. E. Wisniewski, W. Liu, C. E. Whiteford, M. Conde, S. Doran, C. Jing et al., Overestimation of thermal emittance in solenoid scans due 
to coupled transverse motion, Phys. Rev. Accel. Beams 21, 122803 (2018).

[66] E. Wisniewski, W. Liu, C. Whiteford, S. Antipov, M. Conde, C. Jing, W. Gai, D. Doran, and J. Power, in Proceedings of the 6th International Particle Accelerator Conference, Richmond, VA, USA (JACoW, Geneva, Switzerland, 2015), pp. 3283-3285.

[67] J. Power, M. Conde, W. Gai, Z. Li, and D. Mihalcea, in Proceedings of the International Particle Accelerator Conference, Kyoto, Japan (ICR, Kyoto, 2010), pp. 4310-4312.

[68] https://www.bergoz.com/en/ict-bcm-ihr.

[69] M. Peng, J. Shao, C. Jing, E. Wisniewski, G. Ha, J. Seok, M. Conde, W. Liu, J. Power, S. Doran, C. Whiteford, J. Shi, H. Zha, and H. Chen, in Proceedings of the 10th International Particle Accelerator Conference, Melbourne, Australian (JACoW, Geneva, Switzerland, 2019).
[70] W. Wuensch, A. Degiovanni, S. Calatroni, A. Korsbäck, F. Djurabekova, R. Rajamäki, and J. Giner-Navarro, Statistics of vacuum breakdown in the high-gradient and low-rate regime, Phys. Rev. Accel. Beams 20, 011007 (2017).

[71] X. Wu, J. Shi, H. Chen, J. Shao, T. Abe, T. Higo, S. Matsumoto, and W. Wuensch, High-gradient breakdown studies of an X -band Compact Linear Collider prototype structure, Phys. Rev. Accel. Beams 20, 052001 (2017).

[72] The General Particle Tracer (GPT) code, Pulsar Physics, The Netherlands: http://www.pulsar.nl/gpt/.

[73] X. Wang and P. Musumeci, in Report of the Basic Energy Sciences Workshop on the Future of Electron Sources, Menlo Park, CA, USA (DOE, Gaithersburg, MD, 2016).

[74] C. Li, W. Gai, C. Jing, J. Power, C. Tang, and A. Zholents, High gradient limits due to single bunch beam breakup in a collinear dielectric wakefield accelerator, Phys. Rev. Accel. Beams 17, 091302 (2014). 